\title{
Chern-Simons Perturbation Theory
}

\author{
Scott Axelrod \\ Math Department, Harvard University and MIT \\ I.M. Singer \\ Math Department, MIT
}

\begin{abstract}
We study the perturbation theory for three dimensional Chern-Simons quantum field theory on a general compact three manifold without boundary. We show that after a simple change of variables, the action obtained by BRS gauge fixing in the Lorentz gauge has a superspace formulation. The basic properties of the propagator and the Feynman rules are written in a precise manner in the language of differential forms. Using the explicit description of the propagator singularities, we prove that the theory is finite. Finally the anomalous metric dependence of the 2-loop partition function on the Riemannian metric (which was introduced to define the gauge fixing) can be cancelled by a local counterterm as in the 1-loop case [28]. In fact, the counterterm is equal to the Chern-Simons action of the metric connection, normalized precisely as one would expect based on the framing dependence of Witten's exact solution.
\end{abstract}

This work was supported in part by the Divisions of Applied Mathematics of the U. S. Department of Energy under contracts DE-FG02-88ER25065 and DE-FG02-88ER25066. 


\section{Introduction}

One of the most successful topological quantum field theories considered to date has been the three dimensional Chern-Simons theory with the inclusion of Wilson loops. In his seminal paper [28] and in subsequent work [29], E. Witten described the exact solution for this theory and showed that the observables lead to a broad class of invariants of compact three manifolds with imbedded knots (also with choices of orientations, framings and labelings), generalizing the knot invariants defined by V. Jones [21]. Witten's starting point was a Feynman path integral formulation of the observables. Of course from a mathematical point of view this starting point is purely formal. What Witten did, however, was perform formal manipulations of the path integral, based upon physical insight and experience, to arrive at an ansatz for the solution. Subsequent work by several authors [10], [22], [26], [11] have verified and made rigorous Witten's main results. Also a theory essentially equivalent to Witten's solution of Chern-Simons was described in [24].

When no links are present, the invariant for a compact oriented 3-manifold $M$ (taken here to have no boundary) is given by the partition function

$$
Z_{k}(M, G)=\int \mathcal{D} A e^{i k C S(A)}
$$

The basic field $A$ in Eq. 1.1 is a connection (gauge field) with compact gauge group $G$, and the Chern-Simons action is given by

$$
C S(A)=\frac{1}{4 \pi} \int_{M} \operatorname{Tr}\left(A \wedge d A+\frac{2}{3} A^{3}\right) .
$$

Here $\operatorname{Tr}$ is the basic trace on the Lie algebra $\mathbf{g}$ of $G$ normalized so that the pairing $(A, B)=$ $-\operatorname{Tr}(A B)$ on $\mathbf{g}$ is the basic inner product ${ }^{1}$. For notational simplicity, we have taken the underlying principal bundle to be trivialized and identified the connection $A$ with a $\mathbf{g}$ valued one form on $M$.

Witten's solution follows not by "evaluating" the path integrals directly but by exploiting deep connections with rational conformal field theory. However, the motivation behind and the intuition for the construction is that there is some a priori definition of the path integral which behaves as expected physically. Unfortunately, a direct definition of the full non-perturbative path integral seems beyond the reach of our present-day techniques.

\footnotetext{
1 Normalized so that it corresponds, under the Chern-Weil homomorphism, to a generator of $H^{4}(B \tilde{G}, \mathbb{Z})$, where $\tilde{G}$ is the simply-connected cover of $G$.
} 
Here we study the perturbative formulation. Chern-Simons perturbation theory on flat $\mathbb{R}^{3}$ has been looked at previously by several groups of physicists. In [17], the theory up to 2-loops was found to be finite and to give knot invariants. In [7], [8], [12] a superspace formulation of the gauged fixed action was given. Two different arguments for finiteness to all orders are presented in [8] and [13], both assuming a nice regularization scheme and employing special symmetries of the gauge fixed action to conclude that the $\beta$ function vanishes. In [4], Dror Bar-Natan gave a rigorous treatment of the perturbative definition of knot invariants in $\mathbb{R}^{3}$ up to 2 loops, and showed it agreed with the results expected from Witten's exact solution.

In this paper, we will allow $M$ to be an arbitrary compact, oriented, 3-manifold without boundary and will obtain a succinct description of the l-loop contribution in the language of differential forms which we show is finite directly. Specifically, we perturb about a solution, $A^{(0)}$, of the equations of motion (i.e. a flat connection). We shall assume that $A^{(0)}$ is isolated up to gauge transformations and that the group of gauge transformations fixing $A^{(0)}$ is discrete. Equivalently, we assume that the cohomology of $d^{(0)}$ vanishes, where $d^{(0)}$ is the exterior covariant derivative twisted by $A^{(0)}$ and acting on $\Omega^{*}(M ; \mathbf{g})$, the space of forms with values in the associated adjoint bundle. The differential forms viewpoint instructs us to sum over all particle types before integrating and provides us with a natural point splitting of the propagator on the diagonal. One could say this is the regularization scheme we use.

To define the perturbative expansion, it is necessary to make a choice of gauge fixing. We choose BRS gauge fixing using Lorentz gauge, which depends on a choice of Riemannian metric, $g$, on $M$. The perturbative expansion has the form

$$
Z_{k}\left(M, A^{(0)}, g\right)=Z_{k}^{s c}\left(M, A^{(0)}, g\right) Z_{k+h}^{h l}\left(M, A^{(0)}, g\right)
$$

where $Z_{k}^{s c}$ is the "semi-classical approximation" and $Z_{k+h}^{h l}$ is the sum of the higher order corrections. We have included an ad hoc shift in $k$ here necessary for agreement with Witten's exact solution. We will explain one reason this shift is needed in $\S 6$, although we can offer, at present, no derivation of it. The role of the shift in $k$ in perturbation theory has been the subject of much discussion in the physics literature. An explanation of the problem is given in [1].

The expansion of $Z_{k}^{h l}$ in inverse powers of $k$ is given by

$$
Z_{k}^{h l}=\sum_{V=0,2,4, \ldots}\left(\frac{-i k}{2 \pi}\right)^{-\frac{1}{2} V} I_{V}^{d i s c}\left(M, A^{(0)}, g\right),
$$


where $I_{V}^{d i s c}$ is the sum of the contributions of all Feynman diagrams with $V$ vertices. Equivalently, the expansion can be written as

$$
Z_{k}^{h l}=\exp \left(\sum_{l=2}^{\infty}\left(\frac{-i k}{2 \pi}\right)^{1-l} I_{l}^{\text {conn }}\left(M, A^{(0)}, g\right)\right)
$$

where $I_{l}^{\text {conn }}$ is the sum of the contribution of the connected Feynman diagrams with $l$ loops.

Our results are best summarized by outlining the paper.

In $\S 2$, we give a form of the gauge fixed action which has a simple superspace formulation Eq. 2.17, and describe the superspace Feynman rules.

In $\S 3$, we rewrite the superspace Feynman rules in a succinct way using the language of differential forms. We describe the basic propagator as a two form on $M \times M$ with values in $\mathbf{g} \otimes \mathbf{g}$ and state its salient properties (PL1)-(PL6). $I_{V}^{\text {disc }}$ will be written as an integral over $M^{V}$ of a top form obtained from the propagator.

In $\S 4$, we sketch the proof of finiteness, in particular that the multiple integral over $M^{V}$ defining $I_{V}^{\text {disc }}$ converges despite the singularity of the propagator on the diagonal. The logic of the proof is as follows. By a fairly general argument, the proof reduces to showing convergence of diagrams for the flat space theory which may also have insertions of edges with a propagator given by a subleading term in the singularity of the curved space propagator. By the convergence theorem, it thus suffices to show that any such flat space diagrams which are superficially divergent vanish. The latter follows by a simple symmetry and power counting argument.

In $\S 5$, we give a formal proof that $I_{V}^{d i s c}\left(M, A^{(0)}, g\right)$ is independent of the metric $g$. By that we mean, a proof that uses integration by parts ignoring the singularities. For the case of two loops, we give a careful treatment using Stoke's theorem. We find an explicit anomaly given as a local integral over $M$ of the form one would expect from power counting and symmetry considerations. The overall coefficient of the anomaly agrees with what one would predict from Witten's exact solution. This means that we obtain a manifold invariant by subtracting a concrete counterterm from $I_{2}^{\text {disc }}=I_{2}^{c o n n}$.

In $\S 6$, we make some comments about the probable relation of the results here to Witten's exact solution and about possible extensions of our results.

A more detailed exposition is in preparation [3]. In it will be found the derivation of the singular behaviour of the propagator near the diagonal and a careful discussion of signs and symmetry factors. 


\section{Superpace Form of Gauge Fixed Action and Feynman Rules}

In this section we derive a form of the gauge fixed action which has a superspace formulation. In fact, the gauge fixed action will be seen to have the same form as the original Chern-Simons action, but applied to a superfield with certain constraints. We will first perform standard BRS gauge fixing in the Lorentz gauge, and then will change variables and integrate out the field multiplying the gauge fixing condition. We arrive at a form of the gauge fixed action which may be written in superspace.

Expanding around $A^{(0)}$, the Chern-Simons action takes the form

$$
C S\left(A^{(0)}+A\right)=C S\left(A^{(0)}\right)+\frac{1}{4 \pi} \int_{M} \operatorname{Tr}\left(A \wedge d^{(0)} A+\frac{1}{3} A \wedge[A, A]\right),
$$

where $A$ is a Lie algebra valued one form.

For the standard gauge fixing, we introduce Fermionic fields $c$ and $\bar{c}$ and a Bosonic field $b$, all valued in the Lie algebra of the group of gauge transformations, $\Omega^{0}(M ; \mathbf{g})$. The BRS operator $Q$ is given by

$$
\begin{aligned}
Q A=-d^{(0)} c-[A, c], & Q c & =\frac{1}{2}[c, c] \\
Q \bar{c}=b, & Q b & =0 .
\end{aligned}
$$

The operator $d^{(0)}$ is the direct sum of operators $d_{q}^{(0)}: \Omega^{q}(M ; \mathbf{g}) \rightarrow \Omega^{q+1}(M ; \mathbf{g})$. To define the Lorentz gauge condition we choose a Riemannian metric $g$ on $M$. This allows us to define the Hodge $*$ operator $*: \Omega^{q}(M ; \mathbf{g}) \rightarrow \Omega^{3-q}(M ; \mathbf{g})$ which satisfies $*^{2}=1$. We choose the sign of $*$ so that the inner product

$$
<\chi, \psi>\equiv-\int_{M} \operatorname{Tr}(* \chi \wedge \psi), \quad \text { for } \chi, \psi \in \Omega^{q}(M ; \mathbf{g})
$$

is positive. Relative to this inner product, the adjoint of $d_{q}^{(0)}$ is $\left(d_{q}^{(0)}\right)^{\dagger}=(-1)^{q+1} * d_{2-q}^{(0)} *$. $d^{(0) \dagger}$ will also be denoted by $\delta^{(0)}$.

The Lorentz gauge condition is $\delta^{(0)} A=0$. This condition is implemented by the gauge fixed action

$$
\begin{aligned}
S_{g f}(A, c, \bar{c}, b) & =k C S\left(A^{(0)}+A\right)+Q V \\
V & =\alpha<\bar{c}, \delta^{(0)} A>,
\end{aligned}
$$

where $\alpha$ is a constant which we are free to select. Choosing $\alpha=k / 2 \pi$, and defining $\mathcal{C}=* d^{(0)} \bar{c}$ and $\mathcal{B}=* d^{(0)} b$, we find

$$
\begin{aligned}
& S_{g f}(A, c, \bar{c}, b)-k C S\left(A^{(0)}\right) \\
& \quad=\frac{k}{2 \pi} \int_{M} \operatorname{Tr}\left(\frac{1}{2} A \wedge d^{(0)} A+\frac{1}{6} A[A, A]-\mathcal{C} \wedge d^{(0)} c-\mathcal{C} \wedge[A, c]-\mathcal{B} \wedge A\right) .
\end{aligned}
$$


By acyclicity of $A^{(0)}$ and elementary Hodge theory, the change of variable from $(b, \bar{c})$ to $(\mathcal{B}, \mathcal{C})$ is an invertible map from pairs of elements of $\Omega^{0}(M ; \mathbf{g})$ to pairs of elements of $\operatorname{Ker}\left(\delta_{1}^{(0)}\right)$. Note that the Jacobian for this change of variable is 1 because the Fermionic determinant for the change from $\bar{c}$ to $\mathcal{C}$ cancels the Bosonic determinant for the change from $b$ to $\mathcal{B}$. Also note that the integral over the field $\mathcal{B}$ just imposes the Lorentz gauge constraint $\delta^{(0)} A=0$. Thus the gauge fixed path integral can be written

$$
Z_{k}\left(M, A^{(0)}, g\right)=\int \mathcal{D} A \mathcal{D} c \mathcal{D} \bar{c} \mathcal{D} b e^{i S_{g f}(A, c, \bar{c}, b)}=e^{i k C S\left(A^{(0)}\right)} \int \mathcal{D} A \mathcal{D} c \mathcal{D} C e^{-S(A, c, \mathcal{C})}
$$

where the last integral is over the Bosonic field $A \in \operatorname{Ker}\left(\delta_{0}^{(0)}\right)$ and the Fermionic fields $c \in \Omega^{0}(M ; \mathbf{g})$ and $\mathcal{C} \in \operatorname{Ker}\left(\delta_{1}^{(0)}\right)$, and the (imaginary) action is given by

$$
S(A, c, \mathcal{C})=-\frac{i k}{2 \pi} \int_{M} \frac{1}{2} A^{a} \wedge d^{(0)} A^{a}-\mathcal{C}^{a} \wedge d^{(0)} c^{a}+\frac{1}{6} f_{a b c}\left(A^{a} \wedge A^{b} \wedge A^{c}-6 \mathcal{C}^{a} \wedge A^{b} \wedge c^{c}\right) .
$$

Here we have chosen coordinates on $\mathbf{g}$ corresponding to an orthonormal basis $T_{a}$ (relative to the basic inner product). The (totally antisymmetric) structure constants $f_{a b c}$ of $\mathbf{g}$ are defined by

$$
\left[T_{b}, T_{c}\right]=f_{a b c} T_{a}
$$

To give Eq. 2.12 a superspace interpretation, we need some supermanifold notation. For $V$ a vector space, we let $V_{-}$denote a Fermionic copy of $V$. More generally, for $E$ a vector bundle over a base manifold $N$, we let $E_{-}$denote $E$ but with the fibers considered Fermionic. So functions on $E_{-}$are sections over $N$ of $\Lambda^{*}(E)^{2}$.

Our base supermanifold is $T M_{-}$, that is the tangent bundle to $M$, but with the fibers considered to be Fermionic (and the base still bosonic). A local coordinate system $x^{\mu}$ on the base $M$ determine Fermionic coordinates $\theta^{\mu}$ on the Fibers of $T M_{-}$. The $\theta^{\mu}$ essentially behave like the one forms $d x^{\mu}$. In fact, there is a correspondence between differential forms, $\tilde{a}$, on $M$ and functions, $a$, on $T M_{-}$(i.e. "superfields") given by

$$
\tilde{a}(x)=\sum_{i} a_{\mu_{1} \ldots \mu_{i}} d x^{\mu_{1}} \ldots d x^{\mu_{i}} \leftrightarrow a(x, \theta)=\sum_{i} \theta^{\mu_{1}} \ldots \theta^{\mu_{i}} a_{\mu_{1} \ldots \mu_{i}} .
$$

Under this correspondence, integration of top forms corresponds to integration of superfields in the natural supervolume form on $T M_{-}$, the exterior differential operator $d$ on forms corresponds to the operator $\theta^{\mu} \frac{\partial}{\partial x_{u}}$ on superfields, and wedge product of differential

2 More precisely, Bosonic (Fermionic) functions on $E_{-}$correspond to a choice of a Bosonic (Fermionic) section of $\Lambda^{\text {even }}(E)$ together with a Fermionic (Bosonic) section of $\Lambda^{\text {odd }}(E)$. 
forms corresponds to multiplication of superfields. Care must be taken with the correspondence of products because in the forms language one takes the $d x^{\mu}$ to commute with Fermions, whereas in the superspace language the $\theta^{\mu}$ anti-commute with Fermions ${ }^{3}$.

Now let $\mathcal{A}$ be a Fermionic $\mathbf{g}$ valued superfield on $T M_{-}$. The operators $d^{(0)}$ and $\delta^{(0)}$ on $\mathbf{g}$ valued forms correspond to operators $d^{(0)}$ and $\delta^{(0)}$ on the superfield $\mathcal{A}$. So that we can make contact with Eq. 2.11, we name the component fields of $\mathcal{A}$ as follows,

$$
\mathcal{A}^{a}(x, \theta)=c^{a}(x)+\theta^{\mu} A_{\mu}^{a}(x)+\theta^{\mu} \theta^{\nu} \mathcal{C}_{\mu \nu}^{a}(x)+\theta^{\mu} \theta^{\nu} \theta^{\rho} B_{\mu \nu \rho}^{a}(x) .
$$

Since $\mathcal{A}$ is Fermionic, $c$ and $\mathcal{C}$ are Fermionic and $A$ and $B$ are Bosonic. Observe that the condition $\delta^{(0)} \mathcal{A}=0$ means precisely that $A$ is in $\operatorname{Ker}\left(\delta_{0}^{(0)}\right), \mathcal{C}$ is in $\operatorname{Ker}\left(\delta_{1}^{(0)}\right)$, and $B$ equals zero. (The last fact follows from acyclicity of $A^{(0)}$.) So the set of $\mathcal{A}$ for which $\delta^{(0)} \mathcal{A}$ vanishes is equal to the set of triples $(c, \mathcal{C}, A)$ which are integrated over in Eq. 2.11. By a simple calculation, the action defined in Eq. 2.12 has a superspace formulation:

$$
S(\mathcal{A})=S(c, \mathcal{C}, A)=\lambda \int d X\left[\frac{1}{2} \mathcal{A}^{a}(X)\left(d^{(0)} \mathcal{A}\right)^{a}(X)+\frac{1}{6} f_{a b c} \mathcal{A}^{a}(X) \mathcal{A}^{b}(X) \mathcal{A}^{c}(X)\right]
$$

For convenience in writing down the Feynman rules, we have introduced the symbol $X$ for the coordinates $(x, \theta)$, the abbreviation $d X$ for the volume form $d^{3} x d^{3} \theta$ on $T M_{-}$, and the constant

$$
\lambda=-i k / 2 \pi
$$

In summary, the path integral is given by

$$
Z_{k}(M)=\int_{\left\{\mathcal{A} ; \delta^{(0)} \mathcal{A}=0\right\}} \mathcal{D} \mathcal{A} e^{-S(\mathcal{A})}
$$

where $\mathcal{A}$ is a $\mathbf{g}$ valued Fermionic superfield on $T M_{-}$and the action $S$ is given by Eq. 2.17.

To write the Feynman rules in the superspace formulation, we must take into account two complications not usually present in the derivation of super-Feynman rules [16], [27].

3 Let $a^{(i)}=\theta_{1}^{\mu} \ldots \theta_{i}^{\mu} a_{\mu_{1} \ldots \mu_{i}}$ denote the piece of $a$ of degree $i$ in the $\theta^{\mu}, \tilde{a}^{(i)}$ denote the piece of $\tilde{a}$ which has form degree $i$, and $|a|= \pm 1$ denote the statistics of $a$. Then $\left|a_{\mu_{1} \ldots \mu_{i}}\right|=(-1)^{|a|+i}$. The product of $a$ with another superfield $b$ is

$$
a(x, \theta) b(x, \theta)=\sum_{i, j}-1^{(i+|a|) j} \theta^{\mu_{1}} \ldots \theta^{\mu_{i}} \theta^{\nu_{1}} \ldots \theta^{\nu_{j}} a_{\mu_{1} \ldots \mu_{i}}(x) b_{\nu_{1} \ldots \nu_{j}}(x) .
$$

Thus the superfield $a b$ obtained by superfield multiplication corresponds to the form $\sum_{i, j}(-1)^{i j+|a| j} \tilde{a}^{(i)} \wedge$ $\tilde{b}^{(j)}$. 
The first complication is to deal properly with the constraint $\delta^{(0)} \mathcal{A}=0$. The second complication is to keep careful track of the overall sign in front of each graph. Care must be taken here because the basic superfield is Fermionic and because the operation of integration over the base supermanifold $T M_{-}$and the operator $d^{(0)}$ are Fermionic.

Let

$$
Z_{\text {free }}[\mathcal{J}]=\int_{\mathcal{A} \in \operatorname{Ker}\left(\delta^{(0)}\right)} \mathcal{D} \mathcal{A} e^{\int d X\left[-\frac{\lambda}{2} \mathcal{A}^{a}(X)\left(d^{(0)} \mathcal{A}\right)^{a}(X)+\mathcal{J}^{a}(X) \mathcal{A}^{a}(X)\right]}
$$

be the partition function for the free theory coupled to a source $\mathcal{J}(X) . \mathcal{J}$ is taken to be a Bosonic $\mathrm{g}$ valued superfield, so that the source term in Eq. 2.20 is Bosonic. To keep track of the constraints we introduce the operators $\hat{\pi}_{d}$ and $\hat{\pi}_{\delta}$ on $\mathbf{g}$ valued superfields which orthogonally project onto the image of $d^{(0)}$ and $\delta^{(0)}$, respectively. By acyclicity of $A^{(0)}$ and Hodge theory, we have

$$
\hat{\pi}_{d}+\hat{\pi}_{\delta}=\hat{\delta}^{\mathbf{g}}
$$

where $\hat{\delta}^{\mathbf{g}}$ is the identity operator acting on $\mathbf{g}$ valued superfields. ( $\hat{\delta}$ will denote the identity operator on ordinary superfields.) In order to complete the square in the exponent in Eq. 2.20 and evaluate $Z_{\text {free }}[J]$ in the standard way, we introduce the Fermionic operator $\hat{L}$ which is the "Hodge theory" inverse of $d^{(0)}$. To define it, first let $\hat{L}_{1}: \operatorname{Im}\left(d^{(0)}\right) \rightarrow \operatorname{Ker}\left(d^{(0)}\right)^{\dagger}$ be the inverse of $d^{(0)}$ as a map from the orthocomplement of its kernel to its image. Then $\hat{L}$ is the operator on $\mathbf{g}$ valued superfields obtained by first using $\hat{\pi}_{d}$ to orthogonally project onto $\operatorname{Im}\left(d^{(0)}\right)$ and then applying $\hat{L}_{1}$. This definition is equivalent to the equations

$$
\begin{aligned}
\operatorname{Ker}(\hat{L}) & =\operatorname{Im}\left(d^{(0)}\right)^{\dagger}=\operatorname{Ker}\left(\delta^{(0)}\right) \\
\operatorname{Im}(\hat{L}) & =\operatorname{Ker}\left(d^{(0)}\right)^{\dagger}=\operatorname{Im}\left(\delta^{(0)}\right) \\
d^{(0)} \circ \hat{L} & =\hat{\pi}_{d} \\
\hat{L} \circ d^{(0)} & =\hat{\pi}_{\delta} .
\end{aligned}
$$

Note that this definition of Hodge theory inverse makes sense even if $A^{(0)}$ is not acyclic, useful in generalizing the results here to allow for zero modes. $\hat{L}$ can also be defined by introducing the Laplacian

$$
\nabla=\delta^{(0)} d^{(0)}+d^{(0)} \delta^{(0)}
$$

which is invertible by acyclicity of $A^{(0)}$. The definition Eq. 2.22 is equivalent to

$$
\hat{L}=\delta^{(0)} \circ \nabla^{-1}
$$


Having introduced $\hat{L}$, we may complete the square,

$$
\begin{gathered}
\int d X\left[-\frac{\lambda}{2} \mathcal{A}^{a}(X)\left(d^{(0)} \mathcal{A}\right)^{a}(X)+\mathcal{J}^{a}(X) \mathcal{A}^{a}(X)\right] \\
=\int d X\left[-\frac{\lambda}{2}\left(\mathcal{A}-\left(\lambda^{-1} \hat{L} \mathcal{J}\right)\right)^{a}(X)\left[d^{(0)}\left(\mathcal{A}-\left(\lambda^{-1} \hat{L} \mathcal{J}\right)\right)\right]^{a}(X)\right. \\
\left.+\frac{1}{2}\left(\lambda^{-1} \hat{L} \mathcal{J}\right)^{a}(X) \mathcal{J}^{a}(X)\right]
\end{gathered}
$$

and shift the variable $\mathcal{A}$ in the usual way to obtain

$$
Z_{\text {free }}[\mathcal{J}]=Z_{\text {free }}[0] e^{\frac{1}{2} \int d X\left(\lambda^{-1} \hat{L} \mathcal{J}\right)^{a}(X) \mathcal{J}^{a}(X)} .
$$

Now we want to write $\hat{L}$ as an integral operator with an integral kernel $L(X, Y)$. For any operator $\hat{K}$ on g-valued superfields (either Bosonic or Fermionic), we define the corresponding integral kernel $K$ to be the superfunction on $T M_{-} \times T M_{-}$with values in $\mathrm{g} \times \mathrm{g}$ so that

$$
(\hat{K} \Psi)_{a}(X)=\int d Y K_{a b}(X, Y) \Psi_{b}(Y)
$$

Note that since the operator $\int d Y$ is Fermionic, the integral kernel $K$ will have the opposite statistics to the operator $\hat{K}$.

The kernel $\delta$ for the identity operator $\hat{\delta}$ on superfields, which one might call the "super-delta function", is Fermionic and satisfies

$$
\int d X d Y \delta(X, Y) \Psi(Y) \Phi(X)=\int d X \Psi(X) \Phi(X) .
$$

This implies more generally that

$$
\int d X d Y \delta(X, Y) \Psi(X, Y, Z)=\int d X \Psi(X, X, Z)
$$

for $\Psi$ a function of three variable in $T M_{-}$. Since $d Y d X$ equals $-d X d Y$, the super-delta function is antisymmetric under exchange of $X$ and $Y$. Similarly, $\delta_{a b}^{\mathbf{g}}(X, Y)=\delta_{a b} \delta(X, Y)$ is antisymmetric under simultaneous exchange of $(X, a)$ and $(Y, b)$. One can show likewise that the kernel for $\hat{L}$ is antisymmetric,

$$
L_{a b}(X, Y)=-L_{b a}(Y, X)
$$

as one would expect for a Fermionic propagator. 
Next we write the partition function with interaction in terms of the free partition function with source in the usual way,

$$
Z_{k}=\left.e^{i S\left(A^{(0)}\right)} \exp \left(-\frac{\lambda}{3 !} \int d X f_{a b c} \frac{\partial}{\partial \mathcal{J}^{a}(X)} \frac{\partial}{\partial \mathcal{J}^{b}(X)} \frac{\partial}{\partial \mathcal{J}^{c}(X)}\right) Z_{\text {free }}[\mathcal{J}]\right|_{\mathcal{J}=0}
$$

The expression $\frac{\partial}{\partial \mathcal{J}^{a}(X)}$ appearing here is defined as follows. Given a variation $\delta \mathcal{J}$ of $\mathcal{J}$, we let $\delta_{\delta \mathcal{J}}$ be the operator of differentiation in the direction of $\delta \mathcal{J}$. This acts on functionals $\Omega(\mathcal{J})$ of $\mathcal{J}$. The operator $\frac{\partial}{\partial \mathcal{J}^{a}(X)}$ is then defined by

$$
\delta_{\delta \mathcal{J}} \Omega(\mathcal{J})=\int d X \delta \mathcal{J}^{a}\left[\frac{\partial}{\partial \mathcal{J}^{a}(X)} \Omega(\mathcal{J})\right]
$$

So, for example, we have

$$
\frac{\partial}{\partial \mathcal{J}^{a}(X)} \int d Y \mathcal{J}^{a}(Y) \mathcal{A}^{a}(Y)=\mathcal{A}(X)
$$

Since $\mathcal{J}$ is Bosonic, $\frac{\partial}{\partial \mathcal{J}^{a}(X)}$ is Fermionic, and so $\frac{\partial}{\partial \mathcal{J}^{a}(X)} \int d Y$ equals $-\int d Y \frac{\partial}{\partial \mathcal{J}^{a}(X)}$. Hence

$$
\frac{\partial}{\partial \mathcal{J}^{a}(X)} \mathcal{J}^{b}(Y)=-\delta_{a b} \delta(X, Y)=\delta_{b a} \delta(Y, X)
$$

Expanding out the exponentials in Eq. 2.31 and Eq. 2.26, and writing $\hat{L}$ in terms of the kernel $L$, we find

$$
Z_{k}=\left.Z_{k}^{s c} \sum_{I, V=0}^{\infty} \frac{(-\lambda / 3 !)^{V}}{V !} \frac{\lambda^{-I}}{I !}\left[\left(\Pi_{i=1}^{V} F_{i}\right) \mathcal{L}^{I}\right]\right|_{\mathcal{J}=0}
$$

where

$$
\begin{aligned}
Z_{k}^{s c} & =e^{i k C S\left(A^{(0)}\right)} Z_{\text {free }}[0] \\
F_{i} & =\int d X f_{a^{i} b^{i} c^{i}} \frac{\partial}{\partial \mathcal{J}^{a^{i}}\left(X_{i}\right)} \frac{\partial}{\partial \mathcal{J}^{b^{i}}\left(X_{i}\right)} \frac{\partial}{\partial \mathcal{J}^{c^{i}}\left(X_{i}\right)}, \\
\mathcal{L} & =\frac{1}{2} \int d Y d Z \mathcal{J}^{a}(Y) L^{a b}(Y, Z) \mathcal{J}^{b}(Z) .
\end{aligned}
$$

We may restrict the sum in Eq. 2.35 to $V$ and $I$ satisfying $3 V=2 I$ since all other terms vanish.

To write $\left.\left[\left(\Pi_{i=1}^{V} F_{i}\right) \mathcal{L}^{I}\right]\right|_{\mathcal{J}=0}$ in a form not involving functional derivatives, we consider sources $\mathcal{J}$ of the form

$$
\mathcal{J}^{a}(Y)=\sum_{i=1}^{V} j_{(i)}^{a} \delta\left(Y, X_{j}\right)
$$


Here $j_{(i)}$ is a Fermionic $\mathbf{g}$ valued source at the $i^{t h}$ position. Using

$$
\frac{\partial}{\partial \mathcal{J}^{a}(X)} \mathcal{J}^{b}(Y)=\delta_{a b} \delta\left(Y, X^{i}\right)=\frac{\partial}{\partial j_{(i)}^{a}} \mathcal{J}^{b}(Y),
$$

we find that the correction to the semiclassical approximation is given by

$$
\begin{aligned}
Z_{k}^{h l} & \equiv \frac{Z_{k}}{Z_{k}^{s c}} \\
& =\sum_{3 V=2 I} \frac{\lambda^{V-I}}{(3 !)^{V}(2 !)^{I} V ! I !} \Pi_{i=1}^{V}\left[\int d X_{i} f_{a^{i} b^{i} c^{i}} \frac{\partial}{\partial j_{(i)}^{a^{i}}} \frac{\partial}{\partial j_{(i)}^{b^{i}}} \frac{\partial}{\partial j_{(i)}^{c^{i}}}\right]_{j_{(i)}=0} L_{t o t}^{I},
\end{aligned}
$$

where, for given $V$,

$$
\begin{aligned}
L_{t o t} & =\sum_{i, j=0}^{V} L_{s}\left(X_{i}, j_{(i)}, X_{j}, j_{(j)}\right) \\
L_{s}\left(X_{i}, j_{(i)}, X_{j}, j_{(j)}\right) & \equiv L_{a b}\left(X_{i}, X_{j}\right) j_{(i)}^{a} j_{(j)}^{b} .
\end{aligned}
$$

For the meaning of this when $i$ equals $j$ see Eq. 3.52. $L_{s}$ is the propagator $L$, but rewritten as a Bosonic superfunction on $(T M \oplus \mathbf{g})_{-} \times(T M \oplus \mathbf{g})_{-}$.

At the level of diagrams, Eq. 2.39 means the superspace Feynman rules assign a factor

$$
\int d X\left[f_{a b c} \frac{\partial}{\partial j^{a}} \frac{\partial}{\partial j^{b}} \frac{\partial}{\partial j^{c}}\right]_{j=0},
$$

to a vertex labeled by the point $(X, j) \in[T M \oplus \mathbf{g}]_{-}$, and a propagator $L_{s}\left(X, j, X^{\prime}, j^{\prime}\right)$ on an edge between vertices labeled by $(X, j)$ and $\left(X^{\prime}, j^{\prime}\right)$. These Feynman rules have a superspace formulation on $[T M \oplus \mathbf{g}]_{-}$even though the original action only had $T M_{-}$as the base supermanifold -the Lie algebra directions were not supercoordinates there. In fact, the superfield $\mathcal{A}$ can be viewed as a bosonic superfield $\mathcal{A}(x, \theta, j)=\mathcal{A}^{a}(x, \theta) j_{a}$ on

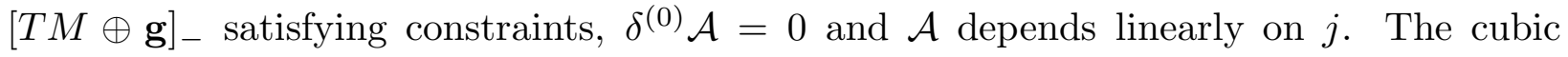
"potential" term in the action Eq. 2.17 can be written as an integral over $[T M \oplus \mathbf{g}]_{-}$, in which Tr becomes a constant superfield. However, the quadratic "kinetic" term cannot be naturally described this way. One sees similar phenomena in other superspace field theories; we hope our techniques will illuminate the special properties of those theories. 


\section{A Closed Form for l-loop Invariants}

In this section we describe the results obtained in the previous section in the language of differential forms rather than superspace. We also summarize in differential forms language the basic properties of the propagator. Since the translation of results from the previous section into the form language here is straight forward, we will not spell it out. Readers not familiar with superspace and gauge fixing can take the statements in this section as a starting point (although we assume some notation from above). Although we do not need it later in the paper, we will use the properties to write the perturbation series in a rather elegant form Eq. 3.57.

We emphasize that the formulation of the Feynman rules given below could have been derived by direct formal manipulations of the path integral (i.e. manipulations which have rigorous analogues for integrals over finite dimensional spaces) without having introduced Fermionic coordinates or even BRS gauge fixing. Such a direct derivation, however, would not explain the simple form of the final answer we derive naturally here.

\section{Properties of the Propagator}

First we describe the basic properties of the propagator $L$. It is the kernel for the operator $\hat{L}=\delta^{(0)} \nabla^{-1}$ acting on $\Omega^{*}(M ; \mathbf{g})$, where $\nabla$ is Laplacian associated to $d^{(0)}$. This means that $L$ is a section of $\Omega^{*}(M \times M ; \mathbf{g} \otimes \mathbf{g})$, which satisfies ${ }^{4}$

$$
(\hat{L} \psi)^{a}(x) \equiv \int_{M_{y}} L_{a b}(x, y) \wedge \psi^{b}(y) .
$$

Note that this definition of $L$ uses wedge products and integration of forms rather than inner products and integration of functions with respect to the Riemannian volume. This will enable us to make metric independence as manifest as possible. The expression $M_{y}$ on the right hand side of Eq. 3.42 is simply an abbreviation to say that we are to integrate over the copy of $M$ paramaterized by the $y$ variable. Since $M$ is 3 dimensional and $\hat{L}$ decreases form degree by 1, Eq. 3.42 implies $L$ is a $3-1=2$ form.

When dealing with products of several copies of $M$ throughout the rest of the paper, we will often adopt the notation used above of distinguishing a particular copy of $M$, and objects associated with it, by adding the name of a variable parameterizing that copy as a subscript. So, for example, we write

$$
L \in \Omega^{2}\left(M_{x} \times M_{y} ; \mathbf{g}_{x} \otimes \mathbf{g}_{y}\right) .
$$

\footnotetext{
4 The unusual sign conventions used in this equation are discussed below.
} 
$L$ is equivalently defined by

$$
\delta_{x}^{(0)} L(x, y)=0, \quad \delta_{y}^{(0)} L(x, y)=0
$$

together with

$$
d_{M \times M}^{(0)} L_{a b}(x, y)=\left(d_{x}^{(0)}+d_{y}^{(0)}\right) L_{a b}(x, y)=-\delta_{a b}^{\mathbf{g}}(x, y) \equiv-\delta_{a b} \delta(x, y)
$$

Here $d_{M \times M}^{(0)}$ is the exterior derivative operator on $\Omega^{*}(M \times M ; \mathbf{g} \times \mathbf{g})$ determined by the connection $\left(A^{(0)}, A^{(0)}\right)$; and $\delta$ is the Poincare dual form to the diagonal, defined by

$$
\int_{M_{x} \times M_{y}} \delta(x, y) \psi(x, y)=\int_{M_{x}} \psi(x, x) \quad \text { for } \psi \in \Omega^{*}(M \times M) .
$$

The fact that $d^{(0) 2}=0$ implies $\hat{L} \circ \hat{L}=0$. Written in terms of the kernel $L$, this becomes

$$
\int_{M_{y}} L_{a b}(x, y) L_{b c}(y, z)=0 \text {. }
$$

Since it is a Fermionic propagator, $L$ is antisymmetric under the involution of $\operatorname{ad}(P) \times$ $\operatorname{ad}(P)$ gotten by exchanging the two copies of $\operatorname{ad}(P)$,

$$
L_{a b}(x, y)=-L_{b a}(y, x)
$$

This can be shown directly from the fact that the involution reverses the orientation of the base $M \times M$, which implies that $\delta(x, y)$ is antisymmetric under exchange of $x$ and $y$. Then, since the involution leaves the operator $d_{M \times M}^{(0)}$ in (PL1) invariant, $L$ must be antisymmetric.

Now we wish to consider how $L$ varies as the metric $g$ changes by an infinitesimal variation $\delta g$. For $K$ an object depending on $g$, we will denote the derivative of $K$ in the direction $\delta g$ by either $\dot{K}$ or $\delta_{\delta g} K$. First notice that properties (PL1) -(PL3) can be stated without reference to the metric $g$. In particular, the right hand side of (PL1) does not vary as one changes the metric. Therefore $d_{M \times M}^{(0)} \dot{L}$ vanishes. But acyclicity of $d^{(0)}$ implies acyclicity of $d_{M \times M}^{(0)}$. So the fact that $\dot{L}$ is closed implies it is exact,

$$
\dot{L} \equiv \delta_{\delta g} L=d_{M \times M}^{(0)} B
$$

Here $B$ is a $\mathbf{g} \times \mathbf{g}$ valued one form on $M \times M$ which is also a one form on the space of metrics (i.e. it depends linearly on $\delta g$ ). 
The next important property of the propagator $L$ is the explicit description of its singularities and discontinuities near the diagonal, which we now describe. For $(x, y)$ in a neighborhood of the diagonal, using the Hadamard parametrix method [18] we find ${ }^{5}$

$$
\begin{aligned}
L(x, y) & =L^{h a d}(x, y)+L^{c o n t}(x, y) \\
L^{h a d}(x, y) & =L^{\operatorname{sing}}(x, y)+L^{b d}(x, y) \\
4 \pi L_{a b}^{\text {sing }}(x, y) & =-\frac{1}{2} \operatorname{det}(g)^{\frac{1}{2}} \epsilon_{\mu \nu \rho} \frac{u^{\mu}}{\|u\|^{3}} \widetilde{d u} \widetilde{d u}^{\rho} \delta_{a b} \\
4 \pi L_{a b}^{b d}(x, y) & =-g_{\mu \nu} \frac{u^{\mu}}{\|u\|} \hat{\mathcal{R}}^{\nu} \delta_{a b} \\
\hat{\mathcal{R}}^{\nu} & =\frac{1}{2} \operatorname{det}(g)^{-\frac{1}{2}} \epsilon^{\nu \rho \sigma} R_{\alpha \beta \rho \sigma} d z^{\alpha} d z^{\beta},
\end{aligned}
$$

where $L^{\text {cont }}$ is smooth away from the diagonal and continuous across the diagonal. Here $z \in M$ and $u \in T_{z} M$ are related to $x$ and $y$ by the exponential map,

$$
(x, y)=\exp _{(z, z)}((u,-u))
$$

The "horizontal" one forms $\widetilde{d u}^{i}$ are defined by

$$
\widetilde{d u}^{\mu}=d u^{\mu}+\Gamma_{\nu \rho}^{\mu} u^{\nu} d x^{\rho}
$$

The quantity $\|u\|$ is the Riemannian norm of $u$. In the third and fourth lines of (PL5), we have identified the Lie algebra at $x$ with the Lie algebra at $y$ using the parallel transport operator determined by $A^{(0)}$ along the short geodesic from $x$ to $y$ (of length $2\|u\|$ ). In [3], we derive (PL5) from the Hadamard construction of the kernel for $\nabla^{-1}$, and also give an alternate derivation using equivariant differential forms.

Note that $L^{\text {sing }}$ diverges quadratically as one approaches the diagonal and $L^{b d}$ is discontinuous but bounded.

For the proof of finiteness in $\S 4$, it will be also be useful to describe the propagator singularities in the following way (which, with some care, can be derived from the above). For $z^{(0)} \in M$ and small $y_{1}, y_{2} \in T_{z^{(0)}} M$, we let $x_{i}=\exp _{z^{(0)}}\left(y_{i}\right), i=1,2$. Then

$$
L_{a b}\left(x_{1}, x_{2}\right)=-\delta_{a b}\left[\epsilon_{\mu \nu \rho} \hat{u}^{\mu} d \hat{u}^{\nu} d \hat{u}^{\rho} \alpha+\epsilon_{\mu \nu \rho} \hat{u}^{\mu} d \hat{u}^{\nu} \gamma^{\rho}\right]+(\text { bounded })_{a b}
$$

where $u=y_{1}-y_{2}, \hat{u}=u /\|u\|, \alpha$ is a bounded function of $y_{1}$ and $y_{2}, \gamma^{\rho}$ is a bounded 1 -form for each $\rho$, and (bounded $)_{a b}$ is a bounded 2 -form for each $a$ and $b$.

5 In our conventions, $R_{\alpha \beta \rho \sigma}=\left(R_{\alpha \beta}\right)^{\tau}{ }_{\sigma} g_{\rho \tau}$, where $\left(R_{\alpha \beta}\right)^{\tau}{ }_{\sigma} d z^{\alpha} d z^{\beta}$ is the curvature tensor thought of as an $\operatorname{End}(T M)$ valued 2 -form. 
One can also derive an explicit description of the singularities of $B$ near the diagonal ${ }^{6}$,

$$
\begin{aligned}
B(x, y) & =B^{\text {had }}(x, y)+B^{\text {cont }}(x, y) \\
B^{\text {had }}(x, y) & =B^{\operatorname{sing}}(x, y)+B^{b d}(x, y) \\
4 \pi B_{a b}^{\operatorname{sing}}(x, y) & =\frac{1}{2} \operatorname{det}(g)^{\frac{1}{2}} \epsilon_{\mu \nu \rho} \frac{u^{\mu}}{\|u\|^{3}} \widetilde{d u}^{\nu}\left(g^{\rho \sigma} \delta g_{\sigma \tau} u^{\tau}\right) \delta_{a b} \\
4 \pi B_{a b}^{b d}(x, y) & =g_{\mu \nu} \frac{u^{\mu}}{\|u\|} \hat{\mathcal{O}}^{\nu} \delta_{a b} \\
\hat{\mathcal{O}}^{\nu} & =\frac{1}{2} \operatorname{det}(g)^{-\frac{1}{2}} \epsilon^{\nu \rho \sigma} \mathcal{O}_{\alpha \rho \sigma} d z^{\alpha} \\
\mathcal{O}_{\alpha \rho \sigma} & =\left(\delta \Gamma_{\alpha \rho \sigma}-\frac{1}{2} \nabla_{\alpha} \delta g_{\rho \sigma}\right) .
\end{aligned}
$$

Note that $B^{\text {sing }}$ diverges linearly as one approaches the diagonal, and $B^{b d}$ is bounded and discontinuous.

\section{Form of the Perturbative Expansion}

Having stated the important properties of the propagator in terms of forms, we will state the precise transcription of Eq. 2.39 and Eq. 2.40 into that language.

Let

$$
\Gamma_{x_{1} \ldots x_{V}}=\Gamma\left(M_{x_{1}} \times \ldots \times M_{x_{V}}, \Lambda^{*}\left(\oplus_{i=1}^{V}\left(\left[T^{*} M_{x_{i}} \oplus \mathbf{g}_{x_{i}}\right]\right)\right)\right)
$$

denote the space of sections of the bundle over $M_{x_{1}} \times \ldots \times M_{x_{V}}$ whose fiber at $\left(x_{1}, \ldots, x_{V}\right)$ is the graded Grassmann algebra generated by one forms $d x_{i}^{\mu}$ and $j_{x_{i}}^{a}, i=1, \ldots, V$, corresponding to bases elements for the cotangent space of $M$ and the adjoint bundle of $P$ at the points $x_{i}$. Multiplication on $\Gamma_{x_{1} \ldots x_{V}}$ is pointwise wedge product. The one forms $j_{x_{i}}^{a}$ will also sometimes be denoted by $j_{(i)}^{a}$. The operation of interior product with $j_{(i)}^{a}$ will be denoted $\frac{\partial}{\partial j_{(i)}^{a}} 7$.

Given an element $A \in \Omega^{*}\left(M_{x} \times M_{y} ; \mathbf{g}_{x} \otimes \mathbf{g}_{y}\right)$ (e.g. the propagator $L$ ), we define $A_{s} \in \Gamma_{x y}$ to be the image of $A$ under the natural injection from $\Omega^{*}\left(M_{x} \times M_{y} ; \mathbf{g}_{x} \otimes \mathbf{g}_{y}\right)$ to

$$
\Gamma_{x y}=\Gamma\left(M_{x} \times M_{y} ; \Lambda^{*}\left(\left[T M_{x} \oplus \mathbf{g}_{x}\right] \oplus\left[T M_{y} \oplus \mathbf{g}_{y}\right]\right)\right) .
$$

Also define $A_{t o t} \in \Gamma_{x_{1}, \ldots, x_{V}}$ by

$$
A_{t o t}\left(x_{1}, \ldots, x_{V}\right)=\sum_{i, j=1}^{V} A_{s}\left(x_{i}, x_{j}\right) .
$$

${ }^{6}$ Here $\Gamma_{\alpha \rho \sigma}=g_{\sigma \tau}\left(\Gamma_{\alpha}\right)^{\tau}{ }_{\sigma}$, where $\left(\Gamma_{\alpha}\right)^{\tau}{ }_{\sigma} d z^{\alpha}$ is the connection one-form for the metric connection.

7 The definition of this requires a trivialization of $\mathbf{g}$, and so can only be defined locally when $\mathbf{g}$ is nontrivial. But the equations below are always valid globally. 
Explicitly, the $A_{s}\left(x_{i}, x_{j}\right)$ appearing here are given by

$$
A_{s}\left(x_{i}, x_{j}\right)=A_{a b}\left(x_{i}, x_{j}\right) j_{(i)}^{a} j_{(j)}^{b} .
$$

Although the propagator $L$ is singular along the diagonal, the singularity is symmetric in the group theory indices, because it is proportional to $\delta_{a b}$. Hence $L_{s}$ extends continuously across the diagonal,

$$
L_{s}\left(x_{i}, x_{i}\right) \equiv L_{a b}^{c o n t}\left(x_{i}, x_{i}\right) j_{(i)}^{a} j_{(i)}^{b} .
$$

(This regularization can be stated in any number of equivalent ways as a point splitting regularization.)

Before going on, we make a rather technical comment about our sign conventions in Eq. 3.42 which is a translation into forms language of conventions built into the superspace language. We adopt the unusual sign convention that the expression $\int_{M_{y}}$ in Eq. 3.42 is defined by

$$
\int_{M_{y}}[\psi(y) \chi(x)] \equiv\left[\int_{M_{y}} \psi(y)\right] \chi(x) \quad \text { for } \chi \in \Omega^{*}\left(M_{x}\right), \psi \in \Omega^{*}\left(M_{y}\right) .
$$

This convention is opposite to the usual mathematical definition (see e.g. [9], p. 61]) and means that the exterior derivative operator anticommutes with $\int_{M_{y}}$ rather than commuting. This corresponds, in the notation of the previous section, to the fact that $d \int d X F(X, Y)=-\int d X d F(Y, X)$. More generally, we define the operator $\int_{M_{x_{i}}}$ on $\Gamma_{x_{1} \ldots x_{V}}$ by stating that for $\psi\left(x_{i}\right)$ in $\Gamma_{i}$ and $\chi\left(x_{1}, \ldots, x_{i-1}, x_{i+1}, \ldots, x_{V}\right)$ in $\Gamma_{x_{1} \ldots x_{i-1}, x_{i+1}, \ldots, x_{V}}$,

$$
\int_{M_{x_{i_{1}}}}\left[\psi\left(x_{i}\right) \chi\left(x_{1}, \ldots, x_{i-1}, x_{i+1}, \ldots, x_{V}\right)\right]
$$

equals

$$
\left[\int_{M_{x_{i}}} \psi\left(x_{i}\right)\right] \chi\left(x_{1}, \ldots, x_{i-1}, x_{i+1}, \ldots, x_{V}\right)
$$

if $\psi$ sits inside $\Omega^{*}(M)$ and equals zero if $\psi$ is of degree one or more in the $j_{(i)}^{a}$. Note that, because we chose the nonstandard convention in Eq. 3.53, in order for the operators $\int_{M_{x_{1}} \times \ldots \times M_{x_{V}}}$ and $\int_{M_{x_{1}}} \ldots \int_{M_{x_{V}}}$ to agree, we must equip $M^{V}=M_{x_{1}} \times \ldots \times M_{x_{V}}$ with the nonstandard orientation (for which $\mu_{V} \ldots \mu_{1}$ is a positive volume form on the product if the $\mu_{i}$ are positive volume forms on the factors). 
Now Eq. 2.39 becomes

$$
\begin{aligned}
Z_{k}^{h l} & \equiv \frac{Z_{k}}{Z_{k}^{s c}} \\
& =\sum_{3 V=2 I} \frac{(-i k / 2 \pi)^{V-I}}{(3 !)^{V}(2 !)^{I} V ! I !} \Pi_{i=1}^{V}\left[\int_{M_{x_{i}}} f_{a^{i} b^{i} c^{i}} \frac{\partial}{\partial j_{(i)}^{a^{i}}} \frac{\partial}{\partial j_{(i)}^{b^{i}}} \frac{\partial}{\partial j_{(i)}^{c^{i}}}\right] L_{t o t}\left(x_{1}, . ., x_{v}\right)^{I} .
\end{aligned}
$$

Let $\operatorname{Tr}_{(i)}$ be the operator $f_{a^{i} b^{i} c^{i}} \frac{\partial}{\partial j_{(i)}^{a^{i}}} \frac{\partial}{\partial j_{(i)}^{b^{i}}} \frac{\partial}{\partial j_{(i)}^{c^{i}}}$, and let $\mathrm{TR}$

$$
\mathrm{TR}: \Gamma_{x^{1}, \ldots, x^{V}} \rightarrow \Omega^{*}\left(M_{x_{1}}, \ldots, M_{x_{V}}\right)
$$

be the composition of the operator $\operatorname{Tr}_{(V)} \ldots \operatorname{Tr}_{(1)}$ followed by the restriction of an element in $\Gamma_{x^{1} \ldots x^{V}}$ to the piece of degree 0 in the $j_{(i)}^{a}{ }^{8}$. Then we can rewrite Eq. 3.54 as

$$
Z_{k}^{h l}=\sum_{3 V=2 I} \frac{(-i k / 2 \pi)^{V-I}}{(3 !)^{V}(2 !)^{I} V ! I !} \int_{M^{V}} \operatorname{TR}\left(L_{t o t}\left(x_{1}, . ., x_{v}\right)^{I}\right)
$$

Although we shall not use it anywhere else in the paper, we rewrite $Z_{k}^{h l}$ in one more way that may be useful in trying to sum the perturbation series. Let

$$
e^{M}=\bigcup_{V=0}^{\infty} M^{V} / S_{V}
$$

where $S_{V}$ is the permutation group of order $V$ acting on $M^{V}$ by exchanging the different copies of $M$. ( $e^{M}$ can be identified with the set of finite subsets of $M$.) Then

$$
Z_{k}^{h l}=\int_{e^{M}} \operatorname{TR}\left(e^{\gamma L_{t o t}}\right)
$$

where $\gamma=\frac{1}{2}(-i k / 2 \pi)^{-\frac{1}{3}}(3 !)^{-\frac{2}{3}}$.

\section{Diagramatic Description}

We now describe Eq. 3.54 in the language of Feynman diagrams. For the remainder of the paper, a Feynman diagram will mean a graph $\mathbf{G}$, all of whose vertices have valency 1,2 , or 3. For a diagram $\mathbf{G}, V_{r}(\mathbf{G})$ will denote the number of $r$-valent vertices, $V(\mathbf{G})=$ $V_{1}(\mathbf{G})+V_{2}(\mathbf{G})+V_{3}(\mathbf{G})$ the total number of vertices, $I(\mathbf{G})$ the number of edges, $C(\mathbf{G})$

8 The map TR encodes the proper sign for the interaction vertices. It is possible to interpret it as a generalized trace. 
the number of connected components, and $l(\mathbf{G})$ the number of loops (the dimension of the first homology group). So we have,

$$
\begin{aligned}
2 I(\mathbf{G}) & =3 V_{3}(\mathbf{G})+2 V_{2}(\mathbf{G})+V_{1}(\mathbf{G}), \text { and } \\
V(\mathbf{G})-I(\mathbf{G}) & =C(\mathbf{G})-l(\mathbf{G}) .
\end{aligned}
$$

When the diagram is clear, we will simply write $V$ for $V(\mathbf{G}), I$ for $I(\mathbf{G})$, etc.

The diagrams we look at should be thought of as diagrams for truncated Greens functions. The number of external legs is defined to be

$$
E(\mathbf{G})=3 V(\mathbf{G})-2 V(\mathbf{G})=V_{2}(G)+2 V_{1}(G)
$$

For example, in Figure 1 the external legs are numbered from 1 to 5 .

We now write down a generalization of the amplitude for a truncated graph $\mathbf{G}$ coupled to sources at the external legs. To do so, we order the vertices and let $x_{i}$ be the name of a variable in $M$ labeling the $i$ 'th vertex. The generalization of a collection of external sources will be an element $\Psi$ of $\Gamma_{x_{1}, \ldots, x_{n}}$. We let $\mathcal{I}(\mathbf{G})$ be the product of the propagators for each of the edges of $\mathbf{G}$. To write this down explicitly, we choose an orientation of the graph and an ordering of the edges. Let $i n(e)($ out $(e))$ be the incoming (outgoing) vertex of the $e$ 'th edge. Then $\mathcal{I}(\mathbf{G})$ is given by

$$
\mathcal{I}(\mathbf{G})\left(x_{1}, \ldots, x_{V}\right)=\Pi_{e=1}^{I} L_{s}\left(x_{\text {in }(e)}, x_{\text {out }(e)}\right)
$$

The amplitude for the graph $\mathbf{G}$ coupled to the source $\Psi$ is

$$
\int_{M_{x_{1}} \times \ldots \times M_{x_{V}}} \operatorname{TR}\left(\mathcal{I}(\mathbf{G})\left(x_{1}, \ldots, x_{V}\right) \Psi\left(x_{1}, \ldots, x_{V}\right)\right)
$$

or, more succinctly, $\int_{M^{V}} \operatorname{TR}(\mathcal{I}(\mathbf{G}) \Psi)$.

As an example, to get the amplitude for Figure 1 with a source $\mathcal{J}_{i}$ flowing in at the $i$ 'th external leg, we take

$$
\Psi\left(x_{1}, x_{2}, x_{3}, x_{4}, x_{5}\right)=\mathcal{J}_{1}\left(x_{1}\right) \mathcal{J}_{2}\left(x_{1}\right) \mathcal{J}_{3}\left(x_{4}\right) \mathcal{J}_{4}\left(x_{4}\right) \mathcal{J}_{5}\left(x_{5}\right)
$$

The Feynman amplitude for a diagram $\mathbf{G}$ with no external legs (i.e. a trivalent graph), is the amplitude when $\Psi$ is equal to 1 ,

$$
I(\mathbf{G}) \equiv \int_{M^{V}} \operatorname{TR}(\mathcal{I}(\mathbf{G}))
$$

For the special case of the empty graph, we set $I(\mathbf{G})=1$. 
In order to write $I(\mathbf{G})$ in a way making the group theory indices explicit, we make the following definition. A labeling of a graph $\mathbf{G}$ is a choice of (i) an ordering of the vertices from 1 to $V$, (ii) an ordering of the edges from 1 to $I$, (iii) an orientation, and (iv) an ordering of the three edges incident on any given vertex. Graphs with labels will be denoted $\overline{\mathbf{G}}$, where $\mathbf{G}$ is the underlying unlabeled graph. Given a labeled graph $\overline{\mathbf{G}}$, one can define an injection

$$
F:\{1, \ldots, I\} \times\{1,2\} \rightarrow\{1, \ldots, 3 V\}
$$

by setting $F(e, 1)=3(i n(e)-1)+j_{\text {in }}(e)$ and $F(e, 2)=3($ out $(e)-1)+j_{\text {out }}(e)$ when the $e$ 'th edge points from the vertex $i n(e)$ to the vertex out $(e)$ and is ordered as the $j_{i n}(e)$ 'th edge incident on $i n(e)$ and the $j_{\text {out }}(e)^{\prime}$ 'th edge incident on out $(e)$. Similarly, such an injection $F$ determines a labeled graph $\overline{\mathbf{G}}$. The map $F$ is onto precisely when the underlying graph $\mathbf{G}$ is closed (has no external legs). It will be convenient to abbreviate $F(e, 1)$ by $e(1), F(e, 2)$ by $e(2)$,

The Feynman amplitude for a closed, labeled diagram $\overline{\mathbf{G}}$ is

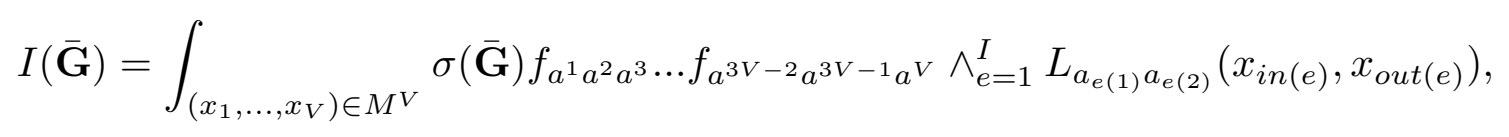

where $\sigma(\overline{\mathbf{G}})$ is equal to \pm 1 . The overall $\operatorname{sign} \sigma(\overline{\mathbf{G}})$ is such that $I(\overline{\mathbf{G}})$ is equal to the Feynman amplitude $I(\mathbf{G})$ defined above. This overall sign is irrelevant for the proof given in the next section that the integral in Eq. 3.65 is convergent despite the singularities near the various diagonals. For the proof of metric independence up to local anomalies, however, the relative signs between graphs are crucial.

The higher loop perturbation series $Z_{k}^{h l}$ which is the focus of our study is given by a weighted sum of the Feynman amplitudes of the labeled trivalent graphs,

$$
Z_{k}^{h l}=\sum_{\overline{\mathbf{G}}} \frac{(-i k / 2 \pi)^{V-I}}{(3 !)^{V}(2 !)^{I} V ! I !} I(\overline{\mathbf{G}})
$$

$Z_{k}^{h l}$ can be rewritten

$$
Z_{k}^{h l}=\sum_{V=0,2, \ldots}^{\infty}(-i k / 2 \pi)^{-\frac{1}{2} V} I_{V}^{d i s c}
$$

where $I_{V}^{\text {disc }}$ is the contribution of all graphs (connected or not) with $V$ vertices,

$$
I_{V}^{\text {disc }}=\sum_{\substack{\overline{\mathbf{G}} \\ V(\mathbf{G})=V}} \frac{1}{(3 !)^{V}(2 !)^{I} V ! I !} I(\overline{\mathbf{G}})
$$


This can also be written as a sum over unlabeled graphs,

$$
I_{V}^{d i s c}=\sum_{\substack{\mathbf{G} \\ V(\mathbf{G})=V}} \frac{1}{S(\mathbf{G})} I(\mathbf{G}),
$$

where $S(\mathbf{G})$ is the symmetry factor of the graph $\mathbf{G}^{9}$.

Letting $I_{l}^{\text {conn }}$ be the contribution of l-loop connected graphs, we have

$$
Z_{k}^{h l}=\exp \left(\sum_{l=0}^{\infty}\left(\frac{-i k}{2 \pi}\right)^{1-l} I_{l}^{c o n n}\right)
$$

\section{Proof of Finiteness}

In this section we sketch the proof of finiteness of Chern-Simons perturbation theory for all $M, A^{(0)}$, and $g$ as above. Further details will appear in [3]. The following strong definition of finiteness implies that the Feynman integrals computing all correlation functions (including the partition function itself) are absolutely convergent. We say that the theory is finite if, for every graph $G$ with vertices labeled by points $x_{1}, \ldots, x_{V}$ and every bounded section $\Psi \in \Gamma_{x_{1}, x_{2}, \ldots, x_{V}}$, the integral $\int_{M^{V}} \operatorname{TR}(\mathcal{I}(\mathbf{G}) \Psi)$ is convergent.

Although we have been considering theories formulated on compact $M$, it also makes sense to formulate perturbation theory for noncompact manifolds. To do so, one should impose conditions at infinity to arrive at an appropriate definition of the propagator, and then use the same formulas as above for the perturbation theory. In particular, for flat $\mathbb{R}^{3}$ with the trivial connection, the propagator is the free propagator,

$$
4 \pi L_{a b}^{f r e e}(x, y)=-\frac{1}{2} \delta_{a b} \epsilon_{\mu \nu \rho} \frac{u^{\mu}}{\|u\|^{3}} d u^{\nu} d u^{\rho}
$$

where $x, y \in \mathbb{R}^{3}$ and $u$ equals $x-y$. We say a theory on a noncompact manifold is ultraviolet finite if the integral $\int_{M^{V}} \operatorname{TR}(\mathcal{I}(\mathbf{G}) \Psi)$ is always locally integrable. (For a compact manifold ultraviolet finiteness is the same as finiteness.)

As a warmup to the general proof of finiteness, we first prove:

9 To define $S(\mathbf{G})$, we let $P_{V, I}$ be the group of order $(3 !)^{V}(2 !)^{I} V ! I$ ! generated by changes of orientation on any of the $I$ edges, and permutations of the set $\{1, \ldots V\}$ of vertex labels, the set $\{1, \ldots, I\}$ of edge labels, and of the orderings of incident vertices on any of the $V$ edges. $P_{V, I}$ acts on the set of labeled graphs with $V$ vertices and $I$ edges. The orbits of the action being sets of labeled graphs with the same underlying unlabeled graph. $S(\mathbf{G})$ is the number of elements of $P_{V, I}$ which fix a labeled graph $\overline{\mathbf{G}}$ with underlying graph $\mathbf{G}$. 
Theorem 4.1 . The theory is ultra-violet finite in flat $\mathbb{R}^{3}$.

Then we shall prove:

Theorem 4.2 . The theory is finite for a general oriented compact 3-manifold $M$ without boundary.

Sketch of Proof of Theorem 4.1.

For a general flat space theory, the superficial degree of divergence, $\Delta(\mathbf{G})$, for a diagram $\mathbf{G}$ is the degree by which one would expect its flat space Feynman integral to diverge due to the singularities when all the vertices approach one another. For the theory we are considering, the superficial degree of divergence of $\mathbf{G}$ is

$$
\Delta(\mathbf{G})=2 I-3(V-C)=3 C-E
$$

That is, one counts plus 2 for each edge because of the $1 /\|u\|^{2}$ divergence of the propagator, minus 3 for the integration at each vertex, and plus 3 since the overall translation collective coordinate for each connected component does not help with convergence. The right hand side of Eq. 4.72 also equals $3 l-I$ as one one would expect from momentum space power counting: plus 3 for the 3 momentum integrated over for each loop and minus 1 because the momentum space propagator falls off as one power of the momentum (since it is the kernel of a differential operator of order 1).

We call a connected diagram superficially divergent if its superficial degree of divergence is non-negative and if it has at least one loop (tree graphs should not be considered divergent). A general diagram is called superficially divergent if any of its connected subdiagrams are.

Recall the convergence theorem for Feynman integrals which says that if a Feynman diagram has no superficially divergent connected subdiagrams then it is absolutely convergent locally ${ }^{10}$.

Thus, to prove Theorem 4.1, it suffices to show that $\mathcal{I}(\mathbf{G})$ vanishes whenever $\mathbf{G}$ is a connected superficially divergent diagram. So let $\mathbf{G}$ be such a diagram, with the vertices labeled by $x_{1}, \ldots, x_{V}$.

10 See [19], §8.1.4 and literature cited therein. We use a version of the theorem easily proved by some slight modifications of the proof described in [19]. The proof there is for four dimensional scalar theories, but easily generalizes to any number of dimensions and any type of particles. The proof in [19] also assumes a massive theory, but that is only to avoid infrared divergences, which do not concern us here (we only need convergence locally). Finally, the theorem in [19] only refers to one-particle irreducible diagrams, but that restriction is easily removed once one defines superficially divergent graphs in general as above. 
Plugging $C=1$ into Eq. 4.72,

$$
\Delta(\mathbf{G})=3-E
$$

Combining this with $l>0$ and some algebra (or pictures), we find that either $V=1$ and $E=1$ or $V>1$ and $E<4$. The case $V=1, E=1$ is trivial because $\mathcal{I}(\mathbf{G})$ is $L_{s}^{\text {free }}\left(x_{1}, x_{1}\right)$, which is zero in flat space. So we may assume $V>1$ and $E<4$.

Now let

$$
v^{(0)}=\sum_{i=1}^{V} x_{i}^{\mu} \frac{\partial}{\partial x_{i}^{\mu}}
$$

and

$$
v^{(\mu)}=\sum_{i=1}^{V} \frac{\partial}{\partial x_{i}^{\mu}} \quad \text { for } \mu=1,2,3
$$

be the vector fields on $\left(\mathbb{R}^{3}\right)^{V}$ generating an overall dilation and overall translations. Note that, the $v^{(\alpha)}, \alpha=0,1,2,3$ are linearly independent as long as the $x_{i}$ are not all equal.

A direct computation shows that interior product with any of these vector fields annihilates the propagator,

$$
i\left(v^{(\alpha)}\right) L_{s}^{\text {free }}\left(x_{i}, x_{j}\right)=0 \quad \text { for } \alpha=0,1,2,3 .
$$

Since $\mathcal{I}(\mathbf{G})$ is a product of propagators between different points, it is also annihilated by interior product with any of the $v^{(\alpha)}$. However, the form $\mathcal{I}(\mathbf{G})$ has degree $2 I=3 V-E$. Thus $\mathcal{I}(\mathbf{G})$ is a form on $\left(\mathbb{R}^{3}\right)^{V}$ of codimension $E$ less than 4 which is annihilated by interior product with four vector fields on $\left(\mathbb{R}^{3}\right)^{V}$, linearly dependent almost everywhere. Hence $\mathcal{I}(\mathbf{G})$ vanishes.

Sketch of Proof of Theorem 4.2.

Since $M^{V}$ is compact, it suffices to show that every $\left(x_{1}^{(0)}, \ldots, x_{V}^{(0)}\right) \in M^{V}$ has an open neighborhood $U$ so that the integral

$$
I_{U} \equiv \int_{U} \operatorname{TR}(\mathcal{I}(\mathbf{G}) \Psi)
$$

is convergent for every bounded $\Psi \in \Gamma_{x_{1}, \ldots, x_{V}}$. We will take $U$ to be of the form

$$
U=\left\{\left(x_{1}, \ldots, x_{V}\right) \in M^{V} ; x_{i}=\exp _{x_{i}^{(0)}}\left(y_{i}\right), y_{i} \in T_{x_{i}^{(0)}} M,\left\|y_{i}\right\|<\epsilon\right\},
$$

where $\epsilon$ will be chosen sufficiently small. For the rest of the proof, $\left(x_{1}, \ldots, x_{V}\right)$ will always be a point in $U$. 
If the $x_{i}^{(0)}$ are all distinct, then $I_{U}$ converges because the propagators are bounded in $U$. Let $z_{1}^{(0)}, \ldots, z_{K}^{(0)}$ be the distinct points in the set $\left\{x_{1}^{(0)}, \ldots, x_{V}^{(0)}\right\}$. By choosing $\epsilon$ small enough, there is some constant $C$ so that the distance between $x_{i}$ and $x_{j}$ is greater than $C$ unless $x_{i}^{(0)}$ equals $x_{j}^{(0)}$. For $J$ between 1 and $K$, let $\mathcal{I}_{J}$ be the product of propagators for edges $e$ connecting vertices close to $z_{J}^{(0)}$, i.e.

$$
\mathcal{I}_{J}=\Pi_{e \in S_{J}} L\left(x_{\text {in }(e)}, x_{\text {out }(e)}\right), \quad S_{J}=\left\{e ; x_{\text {in }(e)}^{(0)}=x_{\text {out }(e)}^{(0)}=z_{J}^{(0)}\right\} .
$$

Then

$$
I_{U}=\int_{U} \operatorname{TR}\left(\left[\Pi_{J=1}^{K} \mathcal{I}_{J}\right] \Psi^{\prime}\right),
$$

where $\Psi^{\prime} \in \Gamma_{x_{1}, \ldots, x_{V}}$ is bounded on $U$. Now $U=U_{1} \times \ldots \times U_{K}$, where $U_{J}$ is the set of positions of the $x_{i}$ for $i$ such that $x_{i}^{(0)}=z_{J}^{(0)}$. Also $\Psi^{\prime}$ can be uniformly approximated by a sum of terms of the form $\Pi_{J=1}^{K} \Psi_{J}$, where the $\Psi_{J}$ are bounded and (as forms) only depend on the variables in $U_{J}$. Consequently, to prove that $I_{U}$ converges, it suffices to show that $\int_{U_{J}} \operatorname{TR}\left(\mathcal{I}\left(\mathbf{G}_{J}\right) \Psi_{J}\right)$ is finite, where $\mathbf{G}_{J}$ is the subgraph of $\mathbf{G}$ consisting of the vertices close to $z_{J}^{(0)}$ and the edges connecting two such vertices (so $\mathcal{I}_{J}$ above equals $\mathcal{I}\left(G_{J}\right)$ ). Replacing $G$ above by $G_{J}$, we can assume that $K=1$.

To recapitulate, it suffices to show convergence of

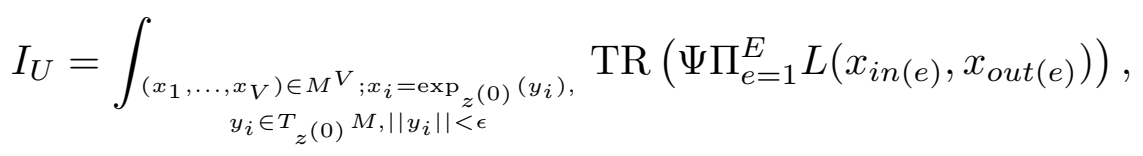

for any graph $\mathbf{G}$ and any bounded $\Psi$. Now use the exponential map to pull back the integral in Eq. 4.80 to an integral over the $y_{j}$ 's; choose a basis $\left\{e_{\mu}\right\}$ of $T_{z^{(0)}} M$; and substitute the expression in Eq. 3.47. We obtain

$$
I_{U}=\int_{\substack{\left(y_{1}, \ldots, y_{V}\right) ; \\ y_{i} \in T_{z}(0) M,\left\|y_{i}\right\|<\epsilon}} \operatorname{TR}\left(\Psi \Pi_{e=1}^{I}\left[B_{\mu \nu}^{e} d\left(\hat{u}_{e}\right)^{\mu} d\left(\hat{u}_{e}\right)^{\nu}+C_{\mu}^{e} d\left(\hat{u}_{e}\right)^{\mu}+D^{e}\right]\right)
$$

where

$$
\hat{u}_{e}=\frac{y_{\text {in }(e)}-y_{\text {out }(e)}}{\left\|y_{\text {in }(e)}-y_{\text {out }(e)}\right\|}
$$

and the $B$ 's, $C$ 's, and $D$ 's are all bounded. The right hand side of Eq. 4.81 is a sum of terms of the form $\int P \wedge \omega$, where $\omega$ is a bounded form and $P$ is a product of the $d\left(\hat{u}_{e}\right)^{\mu}$.

We can think of $P$ as the Feynman integrand, $\mathcal{I}(\Gamma)$, for a graph $\Gamma$ in a theory with three different types of propagators which can be attached to an edge $e$ connecting $y_{\text {in }(e)}$ to $y_{\text {out }(e)}$, namely the $d\left(\hat{u}_{e}\right)^{\mu}$ for $\mu=1,2,3$, The vertex interaction is wedge product followed 
by integration of top forms. The graph $\Gamma$ may have vertices of valency greater than three and is not allowed to have any edges connecting a vertex to itself.

By the convergence theorem, it suffices to prove that if $\Gamma$ is a superficially divergent connected diagrams then its Feynman integrand $\mathcal{I}(\Gamma)$ vanishes. Now, the degree of divergence of any of the propagators $d\left(\hat{u}_{e}\right)^{\mu}$ is one, as is its form degree. So the degree of divergence of $\Gamma$ is $E(\Gamma)-3(V(\Gamma)-1)$. Thus $\Gamma$ is superficially divergent if $3 V(\Gamma)-E(\Gamma) \leq 3$ (and it has at least one loop). But $E(\Gamma)$ is also the form degree of $\mathcal{I}(\Gamma)$. If $\Gamma$ is superficially divergent, $\mathcal{I}(\Gamma)$ is a form of codimension less than 4. However, $\mathcal{I}(\Gamma)$ is annihilated by interior product with the four vector fields generating overall dilations and translations (which are linearly independent when $V(\Gamma)>1$ as is the case for a diagram with at least one loop and no tadpoles). Thus $\mathcal{I}(\Gamma)$ vanishes if $\Gamma$ is superficially divergent.

\section{Formal Proof of Metric Independence and 2-loop Anomalies}

In this section we discuss the dependence of $I_{V}^{\text {disc }}\left(M, A^{(0)}, g\right)$ on the metric $g$. We give a formal proof that $I_{V}^{d i s c}$ is independent of $g$; i.e., we show that the derivative $\delta_{\delta g} I_{V}^{\text {disc }}\left(M, A^{(0)}, g\right)$ vanishes for an arbitrary variation $\delta g$ of the metric $g$. Our argument is formal; later in this section we compute $\delta_{\delta g} I_{2}^{\text {disc }}$ rigorously and show that it is a local anomaly.

Formal Metric Independence

Let $\dot{I}_{V}^{d i s c}$ denote $\delta_{\delta g} I_{V}^{d i s c}\left(M, A^{(0)}, g\right)$. Using integration by parts formally, we find

$$
\begin{aligned}
\dot{I}_{V}^{d i s c} & =\delta_{\delta g} c_{V} \int_{M^{V}} \operatorname{TR}\left(L_{t o t}^{I}\right) \\
& =c_{V} I \int_{M^{V}} \operatorname{TR}\left(\dot{L}_{t o t} L_{t o t}^{I-1}\right) \\
& =c_{V} I \int_{M^{V}} \operatorname{TR}\left(\left(d B_{t o t}\right) L_{t o t}^{I-1}\right) \\
& =c_{V} I \int_{M^{V}} \operatorname{TR}\left(B_{t o t} d\left(L_{t o t}^{I-1}\right)\right) \\
& =-c_{V} I(I-1) \int_{M^{V}} \operatorname{TR}\left(B_{t o t} \delta_{t o t}^{\mathbf{g}} L_{t o t}^{I-2}\right) \\
& =-c_{V} I(I-1) \int_{M^{V}} \operatorname{TR}\left(\delta_{t o t}^{\mathbf{g}} B_{t o t} L_{t o t}^{I-2}\right)
\end{aligned}
$$

where $c_{V}=\left[(3 !)^{V}(2 !)^{I} V ! I !\right]^{-1}$. Here, we have used

$$
\begin{aligned}
d L_{t o t} & =-\delta_{t o t}, \text { and } \\
\dot{L}_{t o t} & =d B_{t o t},
\end{aligned}
$$


which follow from (PL1) and (PL4).

In Eq. 5.84,

$$
\delta_{t o t}^{\mathbf{g}}=\sum_{i \neq j} \delta\left(x_{i}, x_{j}\right) \delta_{a b} j_{(i)}^{a} j_{(j)}^{b} .
$$

Note that the terms with $i=j$ in Eq. 5.85 vanish because $\delta_{a b}$ is symmetric and the $j$ 's are Fermionic.

To show that the last expression in Eq. 5.83 vanishes, it is perhaps most expeditious to describe the basic cancellations in terms of diagrams. The last expression in Eq. 5.83 is equal to a sum over labeled trivalent graphs with a $\delta^{\mathbf{g}}$ propagator on the first edge, a $B$ propagator on the second edge, and an $L$ propagator on all the other edges;

$$
\dot{I}_{V}^{d i s c}=-\sum_{\overline{\mathbf{G}}} c_{V} \int_{M^{V}} \operatorname{TR}\left(\delta_{s}^{\mathbf{g}}\left(x_{i n(1)}, x_{\text {out }(1)}\right) B_{s}\left(x_{i n(2)}, x_{\text {out }(2)}\right) \Pi_{e=3}^{V} L_{s}\left(x_{i n(e)}, x_{\text {out }(e)}\right)\right) .
$$

This can be rewritten as a sum, with suitably combinatorial factors, of Feynman amplitudes for trivalent graphs with two marked edges. The amplitude is the same as that for an unmarked graph, except one is to make an insertion of a $\delta^{\mathbf{g}}$ (rather than the propagator $L)$ on one marked edge and an insertion of a $B$ on the other marked edge. Now, if the $\delta^{\mathbf{g}}$ edge connects the points $y$ and $z$, then by integrating out the $\delta$ function, we find an amplitude which corresponds to a graph with a four valent vertex inserted. One obtains, in this manner, all diagrams with one four valent vertex and one $B$ edge (with the remaining vertices trivalent and the remaining edges unmarked). In fact, each diagram of this type is obtained in three different ways. So, the Feynman rule associated to the four-valent vertex will be a sum of three terms. Figure 3 illustrates the situation. In the equations below, we choose to write amplitudes with Lie algebra indices explicit, rather than imbedded within form notation. So in Figure 3 we have written the Lie algebra indices that will be used in the equations below as well as the names of the positions of the vertices.

The shaded region in each of the diagrams depicted in Figure 3 is the same except for the location of the external legs. Let $W^{\text {cdef }}\left(x_{1}, x_{2}, x_{3}, x_{4}\right)$ be the amplitude for this region when the external legs are at arbitrary positions $x_{1}, \ldots, x_{4}$ as in Figure 4 . Then the amplitude for the top left, top middle, and top right diagrams in Figure 3 are, respectively,

$$
\begin{aligned}
& \int_{M_{y} \times M_{z}} f_{a c d} f_{b e f} \delta_{a b} \delta(y, z) W^{c d e f}(y, y, z, z), \\
& \int_{M_{y} \times M_{z}} f_{a c f} f_{b d e} \delta_{a b} \delta(y, z) W^{c d e f}(y, z, z, y), \text { and } \\
& \int_{M_{y} \times M_{z}} f_{a c e} f_{b d f} \delta_{a b} \delta(y, z) W^{c d e f}(y, z, y, z)
\end{aligned}
$$


By a more careful analysis, one can check that the overall combinatorial factors and signs are the same for each of these diagrams. Hence the amplitude for the four vertex diagram (IV) is $\int_{M_{x}} G_{c d e f} W^{c d e f}(x, x, x, x)$, where the effective Feynman rule at the four valent vertex is $G_{c d e f}=f_{a c d} f_{a e f}+f_{a c f} f_{a d e}+f_{a c e} f_{a d f}$. But this vanishes by the Jacobi identity!

For the case $V=2$, the argument above simplifies. We now provide the details, including the precise combinatorial factors and signs. There are only two, 2-loop diagrams, which are both connected. We call them the dumbbell diagram and the sunset diagram and they are illustrated in Figure 2.

The Feynman amplitudes, with the correct signs and symmetry factors, are:

$$
I_{\text {dumbbell }}=-\frac{1}{8} \int_{M_{x^{1} \times M_{x^{2}}}} f_{a^{1} b^{1} c^{1}} f_{a^{2} b^{2} c^{2}} L_{a^{1} c^{1}}\left(x_{1}, x_{1}\right) L_{a^{2} c^{2}}\left(x_{2}, x_{2}\right) L_{b^{1} b^{2}}\left(x_{1}, x_{2}\right)
$$

for the dumbbell diagram; and

$$
I_{\text {sunset }}=+\frac{1}{12} \int_{M_{x^{1}} \times M_{x^{2}}} f_{a^{1} b^{1} c^{1}} f_{a^{2} b^{2} c^{2}} L_{a^{1} a^{2}}\left(x_{1}, x_{2}\right) L_{c^{1} c^{2}}\left(x_{1}, x_{2}\right) L_{b^{1} b^{2}}\left(x_{1}, x_{2}\right)
$$

for the sunset diagram. These are the two nonvanishing terms in $c_{2} \int_{M^{2}} \operatorname{TR}\left(L_{t o t}^{3}\right)$. Repeating the derivation of Eq. 5.83 at the diagramatic level, we find

$$
\begin{gathered}
\dot{I}_{\text {dumbbell }}=+\frac{1}{8} \int_{M_{x^{1}} \times M_{x^{2}}} f_{a^{1} b^{1} c^{1}} f_{a^{2} b^{2} c^{2}} B_{a^{1} c^{1}}\left(x_{1}, x_{1}\right) L_{a^{2} c^{2}}\left(x_{2}, x_{2}\right) \delta_{b^{1} b^{2}} \delta\left(x_{1}, x_{2}\right) \\
+ \text { permutations, }
\end{gathered}
$$

and

$$
\begin{gathered}
\dot{I}_{\text {sunset }}=-\frac{1}{12} \int_{M_{x^{1} \times M_{x^{2}}}} f_{a^{1} b^{1} c^{1}} f_{a^{2} b^{2} c^{2}} B_{a^{1} a^{2}}\left(x_{1}, x_{2}\right) L_{c^{1} c^{2}}\left(x_{1}, x_{2}\right) \delta_{b^{1} b^{2}} \delta\left(x_{1}, x_{2}\right) \\
+ \text { permutations. }
\end{gathered}
$$

The permutation terms mean that we should sum over permutations of $B, L$, and $\delta^{\mathbf{g}}$. The terms where the $\delta$ function is placed on the handle of the dumbbell vanish because $\delta_{a b} f_{a b c}$ vanishes. Collecting equal terms, relabeling indices, and integrating out the delta functions, we find

$$
\dot{I}_{d u m b b e l l}=+\frac{1}{4} \int_{M_{x}} f_{a c d} f_{a e f} B_{c d}(x, x) L_{e f}(x, x),
$$

and

$$
\begin{aligned}
\dot{I}_{\text {sunset }} & =+\frac{1}{2} \int_{M_{x}} f_{a c e} f_{a f d} B_{c d}(x, x) L_{e f}(x, x) \\
& =+\frac{1}{4} \int_{M_{x}}\left[f_{a c e} f_{a f d}-f_{a c f} f_{a e d}\right] B_{c d}(x, x) L_{e f}(x, x) .
\end{aligned}
$$

For the last equality, we used antisymmetry of $L_{e f}(x, x)$ under exchange of $e$ and $f$. By the Jacobi identity, $f_{\text {acd }} f_{\text {aef }}+f_{\text {ace }} f_{\text {afd }}+f_{\text {acf }} f_{\text {ade }}=0$, we conclude that $\dot{I}_{2}=\dot{I}_{\text {dumbbell }}+\dot{I}_{\text {sunset }}$ vanishes. 


\section{2-loop Anomalies}

To evaluate $\dot{I}_{V}$ rigorously, we will replace the formal use of integration by parts in Eq. 5.83 by a proper use of Stoke's theorem.

We make the following definitions:

$$
\begin{aligned}
\Delta_{i j} & =\left\{\left(x_{1}, \ldots, x_{V}\right) \in M^{V} ; x_{i}=x_{j}\right\} \text { for } i \neq j \\
\Delta_{t o t} & =\bigcup_{\substack{i, j=1 \\
i \neq j}}^{V} \Delta_{i j}, \text { and } \\
\mathcal{N}_{\epsilon} & =\left\{\left(x_{1}, \ldots, x_{V}\right) \in M^{V} ; d\left(x_{i}, x_{j}\right)<\epsilon \text { for some } i \text { and } j\right\} .
\end{aligned}
$$

Note that when $V=2, \Delta_{12}$ is $\Delta_{t o t}$ and equals the diagonal in $M_{x_{1}} \times M_{x_{2}}$ Moreover, $\mathcal{N}_{\epsilon}$ is isomorphic (by the exponential map) to $N_{\epsilon}$, the ball bundle of radius $\epsilon$ in $T M$. Its boundary, $\partial \overline{\mathcal{N}}_{\epsilon}$ in $M \times M$ is isomorphic to $S_{\epsilon}$, the sphere bundle of radius $\epsilon$ in $T M$.

The finiteness result of $\S 4$ implies

$$
\frac{1}{c_{V}} I_{V}^{d i s c}=\lim _{\epsilon \rightarrow 0} \int_{M^{V}-\mathcal{N}_{\epsilon}} \operatorname{Tr}\left(L_{t o t}^{I}\right)
$$

Hence $^{11}$,

$$
\begin{aligned}
\frac{1}{c_{V}} \dot{I}_{V}^{d i s c} & =\lim _{\epsilon \rightarrow 0} \delta_{\delta g} \int_{M^{V}-\mathcal{N}_{\epsilon}} \operatorname{TR}\left(L_{t o t}^{I}\right) \\
& =\lim _{\epsilon \rightarrow 0} I \int_{M^{V}-\mathcal{N}_{\epsilon}} \operatorname{TR}\left(\dot{L}_{t o t} L_{t o t}^{I-1}\right) \\
& =\lim _{\epsilon \rightarrow 0} I \int_{M^{V}-\mathcal{N}_{\epsilon}} \operatorname{TR}\left(\left(d B_{t o t}\right) L_{t o t}^{I-1}\right) .
\end{aligned}
$$

Now using Stoke's theorem on the manifold $M^{V}-\mathcal{N}_{\epsilon}$, we find

$$
\frac{1}{c_{V}} \dot{I}_{V}^{d i s c}=\lim _{\epsilon \rightarrow 0}\left[I \int_{M^{V}-\mathcal{N}_{\epsilon}} \operatorname{TR}\left(B_{\text {tot }} d\left(L_{\text {tot }}^{I-1}\right)\right)+I \int_{\partial\left(M^{V}-\mathcal{N}_{\epsilon}\right)} \operatorname{TR}\left(B_{\text {tot }} L_{\text {tot }}^{I-1}\right)\right] .
$$

But $d L_{t o t}$ vanishes away from $\Delta_{t o t}$, so the first term on the right hand side of Eq. 5.97 vanishes. Thus we find,

$$
\frac{1}{c_{V}} \dot{I}_{V}^{d i s c}=\lim _{\epsilon \rightarrow 0} I \int_{\partial\left(M^{V}-\mathcal{N}_{\epsilon}\right)} \operatorname{TR}\left(B_{t o t} L_{t o t}^{I-1}\right) .
$$

11 By generalizing the proof of finiteness in $\S 4$, one can prove that the integral $\int_{M^{V}} \operatorname{TR}\left(\dot{L}_{t o t} L_{\text {tot }}^{I-1}\right)$ converges absolutely. This implies that the limits in the last two lines of Eq. 2.31 converge and justifies our exchanging the order of integration and metric variation for the second equality of Eq. 2.31. 
When $V=2$, we obtain

$$
\dot{I}_{2}=3 c_{2} \lim _{\epsilon \rightarrow 0} \int_{(z, u) \in S_{\epsilon}} \operatorname{TR}\left(B_{t o t}(x, y) L_{t o t}^{2}(x, y)\right) .
$$

We remind the reader that the $(z, u)$ coordinates are related to the $x, y$ coordinates by the exponential map Eq. 3.45.

For the next lemma, it is convenient to define bounded pieces of propagators:

$$
\begin{aligned}
L^{b d e d} & =L^{b d}+L^{c o n t}, \text { and } \\
L_{t o t}^{b d e d}(x, y) & =L_{s}(x, x)+L_{s}(y, y)+2 L_{s}^{b d e d}(x, y) .
\end{aligned}
$$

Note also that

$$
\begin{gathered}
L_{t o t}^{\text {sing }}(x, y)=2 L_{s}^{\text {sing }}(x, y) \\
L_{\text {tot }}^{b d}(x, y)=2 L_{s}^{b d}(x, y) .
\end{gathered}
$$

We make similar definitions and observations for $B$.

We now prove

Lemma 5.3 .

$$
\dot{I}_{2}=3 c_{2} \lim _{\epsilon \rightarrow 0} \int_{S_{\epsilon}} \operatorname{TR}\left(L_{t o t}^{s i n g} B_{t o t}^{b d e d} L_{t o t}^{b d e d}\right) .
$$

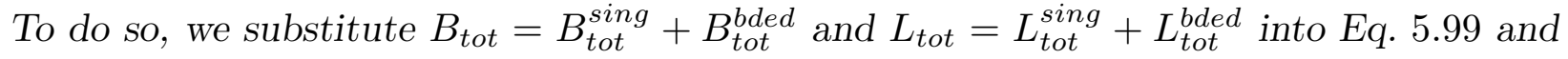
expand the result into eight terms. The term involving only bounded pieces (i.e. only $L_{\text {tot }}^{\text {bded }}$ and $B_{\text {tot }}^{\text {bded }}$ ) vanishes because the integrand is bounded and the measure of the region of integration shrinks to zero as $\epsilon \rightarrow 0$. The terms involving at least two singular pieces vanishes because $\left(L_{\text {tot }}^{\text {sing }}\right)^{2}$ and $L_{\text {tot }}^{\text {sing }} B_{\text {tot }}^{\text {sing }}$ both vanish. (Since they are forms of degree greater than 2 which only depend on the components of $\widetilde{d u}$ in the directions orthogonal to u.) Finally, the quantity $\int_{S_{\epsilon}} \operatorname{TR}\left(B_{\text {tot }}^{\text {sing }} L_{\text {tot }}^{\text {bded }} L_{\text {tot }}^{\text {bded }}\right)$ vanishes in the limit as $\epsilon$ goes to 0 because the volume of the area of the sphere $\left.S_{\epsilon}\right|_{x}$ at each point $x \in M$ shrinks like $\epsilon^{2}$, while $B_{\text {tot }}^{\text {sing }}$ diverges like $1 / \epsilon$, and $L_{\text {tot }}^{\text {bded }}$ remains bounded.

Next, we have

Lemma 5.4 .

$$
\dot{I}_{2}=3 c_{2} \lim _{\epsilon \rightarrow 0} \int_{S_{\epsilon}} \operatorname{TR}\left(L_{t o t}^{s i n g} B_{t o t}^{b d} L_{t o t}^{b d}\right) .
$$


To prove the Lemma, we use Eq. 5.100 and expand Eq. 5.103 into four terms. The Lemma follows from

$$
\begin{aligned}
& 0=\lim _{\epsilon \rightarrow 0} \int_{S_{\epsilon}} \operatorname{TR}\left(L_{\text {tot }}^{\text {sing }} B_{\text {tot }}^{\text {bd }} L_{\text {tot }}^{\text {cont }}\right) \\
& 0=\lim _{\epsilon \rightarrow 0} \int_{S_{\epsilon}} \operatorname{TR}\left(L_{\text {tot }}^{\text {sing }} B_{\text {tot }}^{\text {cont }} L_{\text {tot }}^{\text {bd }}\right) \\
& 0=\lim _{\epsilon \rightarrow 0} \int_{S_{\epsilon}} \operatorname{TR}\left(L_{\text {tot }}^{\text {sing }} B_{\text {tot }}^{\text {cont }} L_{\text {tot }}^{\text {cont }}\right) .
\end{aligned}
$$

Now the operation $\lim _{\epsilon \rightarrow 0} \circ \int_{S_{\epsilon}}$ TR equals the operation $-\int_{M_{z}}$ TR $\circ \lim _{\epsilon \rightarrow 0} \circ \int_{\left.u \in S_{\epsilon}\right|_{z}}$. The minus sign appears because we've taken the non-standard orientation on $M \times M$. So the right hand side of Eq. 5.105.1 equals

$$
-\int_{M_{z}} \operatorname{TR}\left(4 L_{\text {tot }}^{\text {cont }}(z, z) \lim _{\epsilon \rightarrow 0} \int_{u \in S_{\epsilon} \mid z} L_{s}^{\text {sing }}(x, y) B_{s}^{b d}(x, y)\right) .
$$

Substituting the explicit expressions for $L^{\text {sing }}$ and $B^{b d}$ given in (PL5) and (PL6) ${ }^{12}$, Eq. 5.106 equals

$$
\begin{gathered}
-\int_{M_{z}} \operatorname{TR}\left(\sum_{i, j} 4\left[j_{(1)}^{a} j_{(2)}^{a}\right]^{2} L_{t o t}^{c o n t}(z, z)\right)\left[-\frac{1}{2} \epsilon^{\nu \rho \sigma}\left(\delta \Gamma_{\alpha \rho \sigma}(z)-\frac{1}{2} \nabla_{\alpha} \delta g_{\rho \sigma}(z)\right) d z^{\alpha}\right] \\
\lim _{\epsilon \rightarrow 0} \int_{\left.u \in S_{\epsilon}\right|_{z}}\left[\left(-\frac{1}{2} \epsilon_{\beta \gamma \delta} \frac{u^{\beta}}{\|u\|} \frac{d u^{\gamma}}{\|u\|} \frac{d u^{\delta}}{\|u\|}\right) \frac{u^{\nu}}{\|u\|}\right] .
\end{gathered}
$$

The second line in Eq. 5.107 vanishes since it is the integral of a linear function over the two-sphere with its standard volume form. This proves Eq. 5.105.1. The proof of Eq. 5.105 .2 is similar.

Define $L^{\text {sing, } 0}$ so that $L_{a b}^{s i n g}=\delta_{a b} L^{\text {sing,0 }}$ and make similar definitions for $L^{b d, 0}$ and $B^{b d, 0}$. Note that, when restricted to the fibers of $S_{\epsilon}$ above any point in $M, L^{\text {sing, } 0}$ is minus $\Omega$, where $\Omega$ is the spherical volume form of unit area with respect to the standard orientation.

To verify Eq. 5.105.3, we have

$$
\begin{aligned}
\lim _{\epsilon \rightarrow 0} & \int_{S_{\epsilon}} \operatorname{TR}\left(L_{\text {tot }}^{\text {sing }} B_{\text {tot }}^{\text {cont }} L_{\text {tot }}^{\text {cont }}\right) \\
& =-\int_{M_{z}} \operatorname{TR}\left(L_{\text {tot }}^{\text {cont }}(z, z) B_{\text {tot }}^{\text {cont }}(z, z)\left[j_{(1)}^{a} j_{(2)}^{a}\right]\right) \lim _{\epsilon \rightarrow 0}\left[\int_{\left.S_{\epsilon}\right|_{z}} 2 L_{s}^{\text {sing }, 0}\right] \\
& =2 \int_{M_{z}} \operatorname{TR}\left(L_{\text {tot }}^{\text {cont }}(z, z) B_{\text {tot }}^{\text {cont }}(z, z)\left[j_{(1)}^{a} j_{(2)}^{a}\right]\right) .
\end{aligned}
$$

Finally, after unraveling all the notation, the last term in Eq. 5.108 vanishes by the formal proof of metric independence.

12 We use orthonormal coordinates on $T_{z} M$ so that $g_{i j}=\delta_{i j}$. 


\section{Theorem 5.5 .}

$$
\dot{I}_{2}=-\left(\frac{h \operatorname{dim}(G)}{24}\right) \frac{1}{8 \pi^{2}} \int_{M_{z}} \delta \Gamma_{\alpha \rho}^{\nu} R_{\beta \gamma}{ }^{\rho}{ }_{\nu} d z^{\alpha} d z^{\beta} d z^{\gamma}
$$

where $h$ equals the dual Coxeter number of $G$.

In the basis of $\mathbf{g}$ we have chosen, $h$ is given by $2 h \operatorname{dim}(G)=f_{a b c} f_{a b c}$.

Substituting Eq. 5.102 int Eq. 5.104 and performing the TR operation, we find

$$
\dot{I}_{2}=-\frac{1}{4} f_{a b c} f_{a b c} \int_{S_{\epsilon}} L^{s i n g, 0}(x, y) B^{b d, 0}(x, y) L^{b d, 0}(x, y)
$$

Now substitute in the explicit expressions for $L^{s i n g}, L^{b d}$, and $B^{b d}$ and perform the integral over the sphere:

$$
\begin{aligned}
\dot{I}_{2} & =\frac{h \operatorname{dim}(G)}{8(2 \pi)^{2}} \int_{M_{z}} \lim _{\epsilon \rightarrow 0} \int_{\left.u \in S_{\epsilon}\right|_{z}} \Omega\left(g_{\mu \nu} \frac{u^{\mu}}{\|u\|} \hat{R}^{\nu}\right)\left(g_{\rho \sigma} \frac{u^{\rho}}{\|u\|}\right) g_{\rho \sigma} \hat{\mathcal{O}}^{\sigma} \\
& =\frac{h \operatorname{dim}(G)}{24(2 \pi)^{2}} \int_{M_{z}} \hat{R}^{\mu} \hat{O}^{\nu} g_{\mu \nu} .
\end{aligned}
$$

The final expression equals is a sum of two terms: the first term is the right hand side of Eq. 5.109; the second term vanishes by integration by parts and the Bianchi identity.

Let $C S_{\text {grav }}(g, s)$ be the Chern-Simons action for the metric connection associated to $g$ defined using a framing $s$ of the manifold $M$ and normalized using minus half the trace in the adjoint representation of $S O(3)$. (By framing we mean a homotopy class of trivializations of the tangent bundle of $M$.) Since

$$
\delta_{\delta g} C S_{g r a v}(g, s)=-\frac{1}{4 \pi} \int_{M_{z}} \delta \Gamma_{\alpha \rho}^{\nu} R_{\beta \gamma}{ }^{\rho}{ }_{\nu} d z^{\alpha} d z^{\beta} d z^{\gamma}
$$

we obtain

Cor. 5.6 . Let $M$ be an oriented compact 3-manifold without boundary. Let $s$ be a framing and $A^{(0)}$ an acyclic flat $G$-connection. Then the quantity

$$
\tilde{I}_{2}=I_{2}\left(M, A^{(0)}, g\right)-\frac{h \operatorname{dim}(G)}{24} \frac{1}{2 \pi} C S_{g r a v}(g, s)
$$

is independent of the metric $g^{13}$.

13 We warn the reader that we are not confident of the minus sign in the Corollary because of the number of sign conventions used in its derivation. 


\section{Concluding Remarks}

\section{Shift in $k$ and Higher Loop Anomalies.}

Having described a closed form for the l-loop contribution, and having found a manifold invariant at 2 loops, we discuss the relation between our perturbative results so far with Witten's exact solution. For a manifold $M$ with framing $s$, the exact solution will be denoted by $Z_{k}^{\text {exact }}(M, s)$.

Recall Witten's analysis of the semiclassical approximation, $Z^{s c}$. Expanding around a flat acyclic connection $A^{(0)}$, he found that

$$
Z^{s c}\left(M, A^{(0)}, g\right)=\tau\left(M,\left[A^{(0)}\right]\right)^{\frac{1}{2}} e^{\frac{i \pi|G|}{4} \eta_{g r a v}(M, g)} e^{i(k+h) C S\left(A^{(0)}\right)},
$$

where $\tau\left(M,\left[A^{(0)}\right]\right)$ is the analytic torsion of the flat connection $A^{(0)}$ on $M$ [23], [25], and $\eta_{\text {grav }}(M, g)$ is the $\eta$ invariant of the curl operator on $M^{14}$.

As it stands, $Z^{s c}$ is not independent of the metric $g$. However, from [2], one finds

$$
\delta_{\delta g} \eta_{\text {grav }}(M, g)=-\frac{1}{6 \pi} \delta_{\delta g} C S_{\text {grav }}(g, s),
$$

for any framing $s$ Thus, by adding a local counterterm $|G| / 24 C S_{\text {grav }}(g, s)$ to the classical action, one obtains an invariant

$$
\tilde{Z}^{s c}\left(M, A^{(0)}, s\right)=Z^{s c} e^{\frac{i|G|}{24} C S_{\text {grav }}(g, s)}
$$

of framed manifolds with flat acyclic connection. Note that the counterterm added is, up to a constant, the counterterm added in Eq. 5.113 to get a 2-loop invariant.

One expects that the leading asymptotics for large $k$ of Witten's exact solution will reproduce the semi-classical approximation once the counterterms above have been included. More specifically, one expects that, asymptotically for large $k$,

$$
Z_{k}^{\text {exact }}(M, s) \sim \sum_{A^{(0)}} \tilde{Z}_{k}^{s c}\left(M, A^{(0)}, s\right) .
$$

This has been confirmed for many examples [14], [20], [15] ${ }^{15}$. Also, as observed in [28], the behaviour of $Z_{k}^{s c}$ and $Z_{k}^{\text {exact }}$ under a change of framing are consistent with Eq. 6.117:

$$
\begin{aligned}
Z_{k}^{\text {exact }}(M, s+1) & =Z_{k}^{\text {exact }}(M, s) e^{\frac{2 \pi i}{24} \frac{k}{k+h}|G|} \\
\tilde{Z}_{k}^{s c} & =\tilde{Z}_{k}^{s c} e^{\frac{2 \pi i}{24}|G|}
\end{aligned}
$$

14 We normalize $\eta$ invariants as in [2]. The $\eta$ invariants in [28] are smaller by a factor of two.

15 In these examples, some of the flat connections have non-zero betti numbers $\beta_{i}$. In that case, there is an extra factor $(k+h)^{\frac{1}{2}\left(\beta_{1}-\beta_{0}\right)}$ in the definition of $Z_{k}^{s c}$. In order to reproduce the large $k$ asymptotics of the exact solution in these examples, it is also necessary to include a constant factor of one over the order of the center of $G$ whose origin is explained in [30]. 
where we used $C S_{\text {grav }}(g, s+1)=C S_{\text {grav }}(g, s)+2 \pi$.

Eq. 6.117 describes the leading asymptotics of $Z_{k}^{\text {exact }}$. Ignoring for the moment the integration over the moduli space of flat connections, one expects for any nice gauge fixing and regularization procedures that the asymptotics with higher order terms will be given by

$$
Z_{k}^{\text {exact }} \sim \tilde{Z}_{k}^{\text {perp }} \stackrel{\text { def }}{=} \sum_{A^{(0)}} \tilde{Z}_{k}^{s c} \exp \left(\sum_{l=2}^{\infty}\left(-\frac{i k^{\prime}}{2 \pi}\right)^{1-l} \tilde{I}_{l}^{\text {conn }}\right) .
$$

Here $\tilde{I}_{l}$ is the regularized contribution of $l$ loop graphs with a counterterm added to insure metric independence, and $k^{\prime}$ is a function of $k$ whose form depends on the regularization scheme. (There has been much discussion in the physics literature on this point, see for example [1].) The only regularization implicit in this paper was (i) summing over all particle types (BRS ghosts as well as the original dynamical field) before integrating over $M$, and (ii) point splitting regularization of the propagator on the diagonal (i.e. the definition of $\left.L_{s}(x, x)\right)$.

We now argue that $k^{\prime}=k+h$ for our regularization scheme. First, we observe that

$$
I_{l}^{c o n n}\left(-M, A^{(0)}, g\right)=(-1)^{1-l} I_{l}^{c o n n}\left(M, A^{(0)}, g\right)
$$

where $-M$ is the manifold $M$ with the opposite orientation. Next we note that the 2-loop counterterm above changes sign under orientation reversal. We expect counterterms at higher loops to be local and respect the symmetry above, so that

$$
\tilde{I}_{l}^{\text {conn }}\left(-M, A^{(0)}\right)=(-1)^{1-l} \tilde{I}_{l}^{\text {conn }}\left(M, A^{(0)}\right) .
$$

Now let's focus our attention on the case $M=S^{3}$ with $s$ the framing invariant under orientation reversing diffeomorphisms. The exact solution in that case is

$$
Z_{k}^{\text {exact }}=\left(\frac{2}{k+h}\right)^{\frac{1}{2}} \sin \left(\frac{\pi}{k+h}\right) .
$$

Although the only flat connection on $S^{3}$, the trivial connection, is not acyclic, we can still show Eq. 6.121. Thus $\tilde{I}_{l}\left(S^{3}, 0\right)$ vanishes for $l$ even, so that (6.119) implies $k^{\prime}=k+h$.

Next we discuss the specific form of the counterterms, assuming Eq. 6.119 with $k^{\prime}=$ $k+h$. Let us assume that

$$
\tilde{I}_{l}=I_{l}+\beta_{l} C S_{\text {grav }}(g, s)+a_{l}(g),
$$

where $a_{l}(g)$ is a local counterterm independent of any choice of framing and $\beta_{l}$ is a constant. We have already seen that this is the case for $l=1,2$, with $a_{1}=a_{2}=0$. Preliminary 
investigations based on naive power counting support this assumption and even suggest that $a_{l}(g)=0$.

The framing dependence

$$
\tilde{Z}_{k}^{p e r p}(M, s+1)=\tilde{Z}_{k}^{p e r p}(M, s) e^{2 \pi \sum\left(-\frac{i(k+h)}{2 \pi}\right)^{1-l} \beta_{l}}
$$

togeteher with Eq. 6.119 and Eq. 6.118.1 imply that (i) $\beta_{1}=i|G| / 24$. (ii) $\beta_{2}=-h|G| / 24$, and (iii) $\beta_{l}=0$ for $l>2$. Item (i) is the agreement of the framing dependence of the semiclassical approximation with that obtained from the exact solution. Item (ii) says that the framing dependence of our 2-loop invariant given in Cor. 5.6 agrees with that expected from the exact solution. (The caveat to the footnote in Cor. 4.2 holds here as well. Other authors seem to have had as much trouble with sign conventions as we do.) Finally, item (iii) implies that $\beta_{l}=0$. So, we are hopeful that, for $l>2, I_{l}$ is metric independent.

\section{Extensions.}

In the paragraphs above, we discussed the $l$-loop counterterm and the shift from $k$ to $k+h$ which needs to be understood better ${ }^{16}$. We now list other possible extensions of our results and comment on them.

i. One should remove the assumption of acyclicity of $A^{(0)}$. In fact, our proof of finiteness of $I_{l}\left(M, A^{(0)}, g\right)$ remains valid without the assumption of acyclicity. (First, the propagator is still well defined; see the comment after Eq. 2.22. Second, $L^{\text {had }}$ has the same form even when the cohomology doesn't vanish.) However, one must show that the integral of $I_{V}^{\text {disc }}\left(M, A^{(0)}, g\right)$ over the moduli space of flat connections is finite ${ }^{17}$. One knows formally that the variation of $I_{V}^{d i s c}$ is a total divergence, so that its integral is metric independent. This formal proof must be made explicit and rigorous up to local anomalies. Considering the complicated structure of the moduli space, it seems at present a formidable task. We can at least say that Cor. 5.6 holds for $M$ a rational homology sphere and $A^{(0)}$ the trivial connection.

ii. Our results should be extended to include knot invariants. Actually, since we have already shown finiteness of the integral of Greens functions against a bounded source, little needs to be added to show that the terms in the perturbative expansion of Wilson

\footnotetext{
16 A derivation was suggested in a talk by one of us (SA) in May, 1991 at a Conference on "Topological and Geometrical Methods in Field Theory" in Turku, Finland. But evidence against this was subsequently supplied by E. Witten.

17 The integral is relative to $Z_{k}^{s c}$, which still needs to be defined precisely as a measure on the moduli space of flat connections, as opposed to a section of a line bundle.
} 
loop expectation values is finite. The $l$-loop contribution to Wilson loop expectation values can be expressed in terms of the untrunctated Greens functions with $E$ external legs. The latter can be interpreted as elements of $\Omega^{E}\left(M^{E} ; \mathbf{g}^{\otimes E}\right)$. Formally, assuming acyclicity of $A^{(0)}$, these Greens functions are closed, and their derivatives with respect to a metric variation are exact. This will lead to the formal proof of metric independence.

iii. It is natural to try to extend our results to the case when the manifold $M$ has a boundary. Now the exact solution produces a state vector in a Hilbert space associated to the boundary in a nonperturbative way. It is not clear, however, where the perturbative terms belong.

Since the the sewing rule is one of the fundamental properties of a topological field theory reflecting the intuition of path integrals, one would hope to capture some analogue of it at the perturbative level. More modestly, perhaps one could get some feel for factorization perturbatively by trying to prove directly the sewing formula for connected sums:

$$
Z_{k}^{\text {exact }}\left(M_{1} \# M_{2}\right)=\frac{Z_{k}^{\text {exact }}\left(M_{1}\right) Z_{k}^{\text {exact }}\left(M_{2}\right)}{Z_{k}^{\text {exact }}\left(S_{3}\right)} .
$$

That might require computing the perturbative terms for $Z_{k}^{\text {exact }}\left(S_{3}\right)$ (or anything) directly. iv. To reproduce the full exact solution, rather than just its asymptotic behavior to all orders, it would be necessary to sum the perturbation series. Explicit examples seem to indicate that the full partition function is analytic in $\frac{1}{k+h}$ near 0. Eq. 3.57 might be useful for showing this.

v. Finally, it would be of great interest, for example in the application to 3-dimensional gravity, to generalize the results contained here to non-compact gauge groups. Hopefully one could extend the 1-loop analysis of this problem given in [5] to a sensible regularization to all orders in perturbation theory. The manifold invariants thus obtained would have, in our opinion, a rich geometric interpretation in their own right.

Acknowledgements: We would like to thank Edward Witten and Dror Bar-Natan for useful discussions. 


\section{References}

[1] L. Alvarez-Gaume, J.M.F. Labastida, and A.V. Ramallo, A Note on Perturbative Chern-Simons Theory, Nucl. Phys. B334 (1990) 103.

[2] M. Atiyah, V. Patodi and I.M. Singer, Spectral asymmetry and Riemannian geometry I Math. Proc. Camb. Phil. Soc. 77, 43-69 (1975), and II 78 405-432 (1975).

[3] S. Axelrod and I. M. Singer, Perturbative Chern-Simons Quantum Field Theory, in preparation.

[4] D. Bar-Natan, Perturbative Chern-Simons theory, Princeton preprint; Perturbative Aspects of the Chern-Simons Topological Quantum Field Theory, Princeton University Ph.D. Thesis, 1991.

[5] D. Bar-Natan and E. Witten, Perturbative Expansion of Chern-Simons Theory with Non-compact Gauge Group, IAS preprint HEP-91/4.

[6] D. Birmingham and M. Rakowski, Superfield Formulation of Chern-Simons Supersymmetry, Mod. Phys. Lett. A4 (1989) 1753.

[7] D. Birmingham, M. Rakowski, and G. Thompson, Renormalization of Topological Field Theory, Nucl. Phys. B329 (90) 83.

[8] A. Blasi and R. Collina, Finiteness of the Chern-Simons Model in Perturbation Theory, Nucl. Phys. B345 (1990) 472-492.

[9] R. Bott and L.W. Tu, Differential Forms in Algebraic Topology, Springer-Verlag, 1982.

[10] S. Cappell, R. Lee and E. Miller, Invariants of 3-manifolds From Conformal Field Theory, preprint.

[11] L. Crane, 2-D Physics and 3-D Topology, Yale University preprint (1990).

[12] F. Delduc, F. Gieres, and S.P. Sorella, Supersymmetry of the D=3 Chern-Simons Action in the Langau Gauge, Phys. Lett. B225 (1989) 367.

[13] F. Delduc, C. Lucchesi, O. Piguet, and S.P. Sorella, Exact Scale Invariance of the Chern-Simons Theory in the Landau Gauge, Nucl. Phys. B346 (1990) 313-328.

[14] D. Freed and R. Gomph, Computer Calculations of Witten's 3-Manifold Invariant, U. of Texas Preprint.

[15] S. Garoufalidis, Relations Among 3-manifold Invariants, U. of Chicago preprint (1991).

[16] S.J. Gates, JR., M.T. Grisaru, M. Roček, and W. Siegal, Superspace, Benjamin/Cummings, 1983.

[17] E. Guadagnini, M. Martellini, and M. Mintchev, Perturbative Aspects of the ChernSimons Field Theory, Phys. Lett. B277 (1989) 111; Chern-Simons Field Theory and Link Invariants, talk at the 13th Johns Hopkins Workshop on "Knots, Topology, and Field Theory ", Florence, Italy (1989).

[18] L. Hormander, Linear partial differential operators 3 , Springer-Verlag, 1985. 
[19] C. Itzykson and J.B. Zuber, Quantum Field Theory, McGraw-Hill, 1980.

[20] L. Jeffrey, On Some Aspects of Chern-Simons Gauge Theory, Oxford University Ph.D. Thesis, 1991.

[21] V.F.R. Jones, Hecke algebra representations of braid groups and link polynomials, Ann. Math. 126 (1987) 335-388.

[22] R. Kirby and P. Melvin, The 3-Manifold Invariants of Witten and Reshetikhin-Turaev, Berkeley preprint (1990).

[23] D.B. Ray, and I.M. Singer, R-torsion and the Laplacian on Riemannian manifolds, Adv. in Math. 7 (1971) 145-210; Analytic torsion, Proc. Symp. Pure Math 23 (1973) 167.

[24] N. Reshetikhin and V. Turaev, Ribbon Graphs and Their Invariants Derived From Quantum Groups, Comm. Math. Phys 127 (1990) 1-26; Invariants of 3-manifolds via link polynomials and quantum groups, Invent. Math. 103 (1991) 547-597.

[25] A. Schwarz, The Partition Function of Degenerate Quadratic Functionals and RaySinger Invariants, Lett. Math. Phys 2 (1978) 247.

[26] K. Walker, On Witten's 3-manifold Invariants, preprint (1991).

[27] P. West, Introduction ot Supersymmetry and Supergravity, World Scientific, 1986.

[28] E. Witten, Quantum field theory and the Jones polynomial, Comm. Math. Phys. 121 (1989) 351-399.

[29] E. Witten, Gauge Theories, Vertex Models, and Quantum Groups, Nucl. Phys. B330 1990 (286) ; Gauge Theories and Integrable Lattice Models, Nucl. Phys. B322 (1989) 629.

[30] E. Witten, On Quantum Gauge Theories in Two Dimensions, IAS Preprint (1991). 